\title{
Belgeo
}

Revue belge de géographie

$1 \mid 2021$

Miscellaneous

\section{Oran : des terres agricoles sacrifiées pour un urbanisme sauvage}

Oran: agricultural land sacrificed for wild urban development

\section{Hayette Nemouchi et Anissa Zeghiche}

\section{(2) OpenEdition}

1 Journals

Édition électronique

URL : https://journals.openedition.org/belgeo/46093

DOI : $10.4000 /$ belgeo.46093

ISSN : 2294-9135

Éditeur :

National Committee of Geography of Belgium, Société Royale Belge de Géographie

Référence électronique

Hayette Nemouchi et Anissa Zeghiche, «Oran : des terres agricoles sacrifiées pour un urbanisme sauvage », Belgeo [En ligne], 1 | 2021, mis en ligne le 28 mars 2021, consulté le 23 octobre 2021. URL http://journals.openedition.org/belgeo/46093 ; DOI : https://doi.org/10.4000/belgeo.46093

Ce document a été généré automatiquement le 23 octobre 2021.

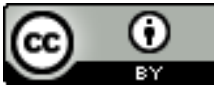

Belgeo est mis à disposition selon les termes de la licence Creative Commons Attribution 4.0 International. 


\section{Oran : des terres agricoles sacrifiées pour un urbanisme sauvage}

Oran: agricultural land sacrificed for wild urban development

Hayette Nemouchi et Anissa Zeghiche

\section{Introduction générale}

\section{Le foncier agricole face au processus d'urbanisation}

1 Pour Lionel Rougé (2018), « la périurbanisation désigne le processus d'extension des agglomérations urbaines, dans leur périphérie, entraînant une transformation des espaces ruraux ». Elle est, aujourd'hui, reconnue comme phénomène mondial animée par différents déterminants (poussée démographique, développement industriel, crise de la ruralité, etc.) et constitue une réponse aux besoins en sols constructibles de plus en plus pressants pour la réalisation des différents plans d'aménagement et de développement des territoires urbains. En effet, les territoires périurbains sont des espaces qui reçoivent l'excédent de la croissance démographique urbaine et qui accueillent les nouveaux projets d'aménagement et de développement (promotions immobilières, implantations de zones d'activités diverses, développement des réseaux de communication, maintien ou développement de certaines agricultures, etc.) en raison de la rareté du foncier disponible en ville. Ils sont, de fait, le lieu et l'expression de recompositions territoriales et de l'évolution des rapports villes-campagne, et plus particulièrement des lieux où s'opèrent de profondes mutations dans tous les registres de l'espace rural (Pérrin et al., 2016 ; Daligaux, 2001 ; Marié, Viard, 1977 ; Peltier, 2010 ; Jarrige et al., 2003 ; Poulot, 2014). Pour Brigitte Prost (1991), les espaces périurbains sont des espaces flous aux marges du système urbain et sont l'expression d'un « marginalisme territorial dans lequel s'affrontent systèmes urbain et rural. Le système rural déstabilisé se désagrège face au système urbain conquérant ", et donc sous une forte emprise de la ville. Ils sont l'arène de jeux d'acteurs et de compétitions pour la 
terre, et de clivage urbain-rural (Darly, Torre, 2008 ; Serrano, Vianey, 2011 ; Houdart et al., 2012 ; Martin et al., 2006 ).

Traiter du foncier agricole n'est pas un exercice aisé au vu des grandes difficultés à surmonter qui sont conceptuelles, méthodologiques et pratiques. Le foncier, ce trait d'union entre l'homme et son milieu, est un objet d'étude complexe, instable et stratégique (enjeux majeurs et sources de convoitise et de conflits). Le foncier est à considérer pas seulement comme un espace ressource et un système d'accès fondé sur le pouvoir de l'accessibilité à la propriété foncière, d'usage et de contrôle des ressources, mais également comme un espace enjeu où le contrôle des ressources et des droits d'accès génèrent des concurrences et des conflits entre les différents secteurs d'activité et entre les différents acteurs et groupes d'acteurs. Il faut reconnaître que ces difficultés relatives à l'étude du foncier agricole sont amplifiées quand elles sont rapportées à un espace périurbain, un espace aux limites floues et évolutives.

Dans les zones périurbaines où se mêlent l'urbain et le rural, les vives tensions autour de la terre s'exacerbent; "en périurbain, l'utilité sociale de la terre est devenue plurielle : l'enjeu du foncier périurbain est de répondre aux besoins d'un territoire 'intermédiaire', un espace d'interface de la ville et de la campagne " (Jouve, Vianey, 2012, p. 27). C'est dire que le foncier a une très forte prégnance sur les configurations et les évolutions des territoires urbains et ruraux et sur les rapports villes-campagnes; l'accès à la ressource foncière par la coopération et par le conflit et l'usage de la ressource sont des indicateurs de la réalité du pouvoir dans la société (Madjarian, 1991, p. 20). Comme le précisent Anne-Marie Jouve et Gisèle Vianey (2012), les meilleures terres agricoles (plaines fertiles) sont détruites par l'urbanisation et l'étalement des infrastructures. Il est courant que la prédation de la ressource foncière agricole par l'urbain s'y exerce sous couvert du droit à la ville et du droit de vivre en ville en prenant la forme de réalisation, en zone périurbaine, d'ensembles résidentiels, d'infrastructures et de zones d'activité économique non agricole pour l'excédent de la population urbaine. Avec l'accélération du processus d'urbanisation, la question foncière est, donc, "une sorte de nœud gordien d'un ensemble de caractères d'une société : elle est à la fois la synthèse des caractères et origines des questions » (Renard, 2003, p. 8).

4 Ainsi, la question de la maîtrise et de la gestion du foncier constitue l'un des dossiers les plus lourds et les plus complexes: si le foncier est la clé de voûte de l'activité socioéconomique; s'il se situe à la croisée des enjeux et des orientations d'aménagement, sa complexité freine souvent la définition des stratégies susceptibles d'y répondre. En somme, parler du foncier, c'est évoquer inévitablement les déterminants historiques, économiques, politiques et idéologiques autour desquels la terre est un enjeu majeur. Le foncier est un objet d'étude stratégique et sensible. Eu ces égards, le foncier ne serait-il pas une boîte noire de la sociologie, de l'économie et de la politique?

5 Dans le contexte algérien, le pouvoir de décider et d'agir aux échelles centrale et locale est presque exclusivement du ressort de l'État et de ses services déconcentrés. Concernant le foncier agricole, il est important de préciser que les terres agricoles des alentours des grandes villes algériennes du nord sont majoritairement propriété de l'État. Elles sont d'anciennes terres coloniales nationalisées au lendemain de l'indépendance et versées au domaine privé de l'État. L'État a, de fait, une mainmise totale sur ce foncier agricole périurbain et toute la latitude d'en disposer à sa guise. Un 
arsenal législatif et juridique a été mis en place pour protéger les terres agricoles contre les détournements d'usage. Dès lors, on pourrait penser que l'État serait le garant de leur protection et de leur préservation via l'application et le respect des règles qu'il a lui-même établies. Mais, dans les faits, la gestion et la maîtrise de cette ressource foncière ne sont pas si évidentes. La pluralité des systèmes d'organisation et d'exploitation (Mutin, 1977; Côte, 1993; Nemouchi, 2009; Zeghiche, 2007), la démultiplication des acteurs, l'enchevêtrement des législations et les objectifs d'aménagement et de développement contradictoires (Omari et al., 2012) ont été autant de facteurs responsables d'une certaine opacité, de confusions et de tensions, de contraintes, de concurrences, voire de nombre de dérives. Ainsi, la politique de protection des terres agricoles est contrariée et chacun des acteurs développe des stratégies foncières pour servir ses intérêts particuliers et agit de manière illicite et plus ou moins désordonnée. C'est dire que les différentes manières dont les acteurs du foncier reçoivent, appliquent, détournent, transforment ou refusent les interventions ou injonctions dont ils ont été l'objet sont des indices révélateurs du degré de complexité, de la forte résonnance de la question foncière et de la difficile articulation entre politiques foncières, politiques urbaines et politiques d'aménagement (Nemouchi, 2009).

6 Aussi, instruire la question foncière en périphérie des grandes métropoles algériennes présente un réel intérêt et invite à explorer des questions sous-jacentes dont celles du rôle du statut juridique des terres agricoles périurbaines dans l'accélération ou le blocage de leur prédation par l'urbain, de la force des instruments d'urbanisme face à cette prédation foncière et de celle des stratégies mises en œuvre pour « légitimer » la dilapidation du foncier agricole périurbain des métropoles algériennes.

7 L'objectif du présent article est de saisir les processus de recomposition de territoires ruraux algériens depuis l'indépendance, d'expliquer les mécanismes d'évolution en œuvre et de décrypter les stratégies développées par les acteurs publics pour transformer les terres agricoles en terres constructibles (terrain à urbaniser ou pour urbanisation futur) dans les espaces périurbains des grandes villes algériennes.

8 Pour donner plus de sens et de réalité aux dynamiques du monde rural algérien, l'investigation porte sur l'espace périurbain d'Oran, deuxième ville d'Algérie et métropole régionale de l'Oranie ; elle vise à apporter quelques éléments d'éclairage sur certains des mécanismes de réappropriation / réaffectation du sol agricole au profit de projets urbains.

9 L'analyse sur le processus de transformation des terres agricoles en terres urbanisées ou à urbaniser est conduite en trois temps. Dans un premier temps, en guise de cadrage, il est entrepris une revue du complexe législatif et institutionnel se rapportant au secteur agricole algérien ; et ce afin d'apprécier la force de l'État de protéger ou non les terres agricoles dont il est le propriétaire contre la machine urbaine. Dans un deuxième temps, l'espace agricole périurbain est resitué face à la puissance hégémonique de la métropole oranaise dans une perspective de montrer l'ampleur des prélèvements de surfaces agricoles au profit de l'urbanisation. La reconstitution spatiotemporelle des surfaces urbanisées dans les espaces périurbains d'Oran s'est appuyée sur l'exploitation des documents graphiques d'urbanisme (PDAU $\left.{ }^{1}, \mathrm{POS}^{2}\right)$, de travaux universitaires réalisés sur Oran et sa périphérie agricole et d'images satellitaires. Dans un troisième temps, il a été mené des enquêtes de terrain. D'une part, nous avons effectué des entretiens semi-directifs au niveau du Groupement Urbain 
d'Oran (GUO) ${ }^{3}$. Nos interlocuteurs sont, principalement, des acteurs ayant un rôle déterminant soit dans la production d'espaces urbanisés (bureaux d'études étatiques en urbanisme URBOR et la Direction de l'Urbanisme et de la construction de la wilaya d'Oran) soit dans les politiques de préservation de l'espace agricole (Direction des Services Agricoles). Ces entretiens ont pour objectif de comprendre comment la question du foncier agricole s'est posée par les praticiens lors de la réalisation des différents documents d'urbanisme (POS, PDAU). En vue d'identifier très concrètement les acteurs qui interviennent sur le foncier et de dévoiler leurs propres stratégies foncières, la commune de Bir El Djir nous a servi d'espace laboratoire. Cette commune est une ancienne localité coloniale de la banlieue oranaise, qui a occupé une position importante dans le processus d'extension du GUO (Bendjelid, 1998). Des entretiens ont été effectués auprès de l'APC ${ }^{4}$ de Bir El Djir et d'un ingénieur aménagiste au Service de l'Urbanisme. Par ailleurs, une enquête à l'échelle de la parcelle (agricole ou îlot du POS) est menée pour rendre compte des actes et stratégies développées localement pour inclure les terres agricoles dans le portefeuille des terres à urbaniser, les mettre sur le marché à bâtir et donc les transformer en ressource locale du projet métropolitain.

\section{Algérie : gouvernance du foncier agricole, choix et perspectives} millions d'hectares de terres pouvant supporter des cultures, des pâturages ou encore des forêts ou des maquis. Ce potentiel naturel correspond à un système écologique et à un espace de production agricole sur lequel se base la sécurité alimentaire du pays. Sur ce total, la superficie agricole utile (SAU) n'est que de 7,5 millions d'hectares, soit $3,14 \%$ de la superficie du territoire national. La gouvernance de ce capital ressource a été et reste le maillon faible dans le processus du développement de l'agriculture algérienne ; les politiques foncières mises en œuvre y ont grandement contribué.

Dès l'indépendance, la majorité des terres agricoles productives coloniales, ceinturant les agglomérations, ont été versées dans le domaine privé de l'État. Cette propriété étatique a fonctionné, d'abord, en Autogestion (Domaines autogérés et CAPAM ${ }^{5}$ ) puis, elle a été agrandie par les terres de propriétaires privés, nationalisés (intégralement ou partiellement) dans le cadre de la Révolution Agraire en 1970. L'État accorde un droit d'usage aux exploitants de ces terres publiques.

Suite aux résultats, jugés insuffisants, des exploitations agricoles du secteur étatique, l'État s'est lancé à plusieurs reprises dans de grandes opérations de restructuration foncière dont les plus importantes sont :

- La réorganisation foncière de 1983 qui visait les anciennes coopératives agricoles de la Révolution Agraire. Ces dernières furent réorganisées sous une nouvelle forme d'unité de production dénommée Domaine Agricole Socialiste (DAS).

- La loi de décembre $1987^{6}$ qui apporta des nouvelles modalités d'exploiter les terres agricoles du domaine privé de l'État. En mettant fin à l'existence des DAS, cette nouvelle loi, appelée aussi la loi 87-19, établit un droit collectif et un droit individuel d'exploitation des terres agricoles. Les anciennes DAS sont, donc, réorganisées en Exploitations Agricoles Collectives (EAC) et Exploitations Agricoles Individuelles (EAI). 
13 Cette succession de réformes et de restructurations foncières a instauré un climat d'instabilité et d'incertitude dans le monde agricole algérien ; l'esprit d'engagement et l'efficience des exploitants agricoles sont profondément affectés. La loi 87/19 prévoyant la perpétuité de la jouissance des terres agricoles du secteur privé de l'État a été modifiée en 2010 par la loi 10-03 du 15 août 20107, réduisant la durée de jouissance d'usage des concessions agricoles à 40 ans. L'insécurité foncière alimentée par une réduction drastique de la durée de jouissance et un certain désengagement de l'État, la précarité du statut des exploitants des terres étatiques, l'émiettement du parcellaire des exploitations ont compromis la mise en valeur agricole et ont encouragé les pratiques informelles telles que le faire-valoir indirect, la location des quotes-parts des EAC, la location ou la vente du matériel agricole acquis grâce à des financements de l'État (Nemouchi, 2011). De telles pratiques révèlent les grandes difficultés de l'État algérien, qui a le droit de propriété sur ces terres agricoles, à maîtriser la gestion du capital foncier agricole et ce malgré ses importants efforts engagés en direction du développement du secteur de l'agriculture.

$14 \mathrm{Au}$ côté de cette situation confuse caractérisant l'organisation interne du secteur agricole, d'autres facteurs externes viennent peser sur l'avenir de l'agriculture. Selon l'ONS, la population algérienne estimée à 42,4 millions d'habitants au $1^{\mathrm{er}}$ janvier 2018 passerait à 51 millions en 2030 et à plus de 70 millions d'habitants à l'horizon 2050 (Bessaoud, 2019). Les implications de cette croissance démographique sont lourdes de significations. Rappelons que l'Algérie est un pays jeune et à forte natalité ${ }^{8}$ signifiant l'importance des besoins actuels et futurs à satisfaire dans tous les domaines de la vie. Le taux d'urbanisation, en Algérie, est passé de $25 \%$ en 1954 à $31,4 \%$ en $1966^{\circ}$, à 70,7 \% en 2015 et atteindrait 82,1\% en 2050 (United Nations, 2014) soit un renversement du ratio rural-urbain et une très forte dominance de l'urbain sur le rural. "En Algérie, sur les côtes comme à l'intérieur du pays, l'urbanisation gagne du terrain: on compte aujourd'hui quatre fois plus de villes qu'il y a un siècle" (Maachou, 2008). Ce phénomène urbain envahisseur gagne des terrains agricoles en créant, ainsi, une pression sur les exploitations agricoles se situant aux abords des grandes et des moyennes agglomérations. Sachant que les développements urbain et rural sont assujettis (l'un comme l'autre) à la mobilisation de la ressource foncière., la réponse à l'explosion démographique, en général, et à la forte concentration urbaine, en particulier, a été de dégager des assiettes foncières, les plus proches des agglomérations urbaines, pour la réalisation de projets d'habitat, d'équipements et d'infrastructures.

Ce choix d'urbaniser les terres agricoles périurbaines a répondu aux soucis d'une économie du moindre coût d'aménagement (exploiter les avantages des sites et des situations favorables) et d'un évitement de contraintes juridiques (cibler le foncier agricole relevant du domaine privé de l'État), peu importe s'il est générateur, aux échelles locales, de conflits d'usage et de droits d'usage. Georges Mutin (1977, p. 582) écrit en référence aux conséquences de la politique nationale de développement des années 1960-1970 sur la déstructuration/désorganisation de l'espace mitidjien « on assiste davantage à une juxtaposition d'actions sectorielles qu'au déploiement d'une véritable d'action régionale» résultant en un total décalage avec les objectifs recherchés. Si réduire à un minimum la prédation du foncier agricole par l'urbanisation constitue un défi majeur des politiques de l'aménagement du territoire en Algérie, les pratiques sur terrain au quotidien viennent contrarier cet objectif. 
Comme partout ailleurs, à Oran les prélèvements de terres agricoles aux fins d'urbanisation, résultant de stratégies individuelles et collectives de contournement juridique, sont générateurs et amplificateurs de conflits d'usage et de droits d'usages entre la ville et l'agriculture périurbaine.

\section{Oran : l'espace agricole périurbain face à la puissance hégémonique de la métropole oranaise}

17 Oran est une ville qui se situe à l'extrémité sud-ouest du bassin méditerranéen, à $450 \mathrm{~km}$ d'Alger, la capitale nationale (fig. 1). Elle est à une demi-heure de vol du port espagnol d'Alicante et d'une heure de Barcelone et de Marseille. Avec ses 1454078 d'habitants en $2008^{10}$, Oran est la $2^{\text {ème }}$ plus grande ville d'Algérie. Elle est le chef-lieu de wilaya du même nom et métropole régionale de l'Oranie. Ville côtière de la région occidentale d'Algérie, elle est un important et dynamique pôle économique et universitaire dont, l'influence s'étend jusqu'aux villes du Sud Algérien.

Figure 1. Localisation et consistance administrative du Groupement Urbain d'Oran.

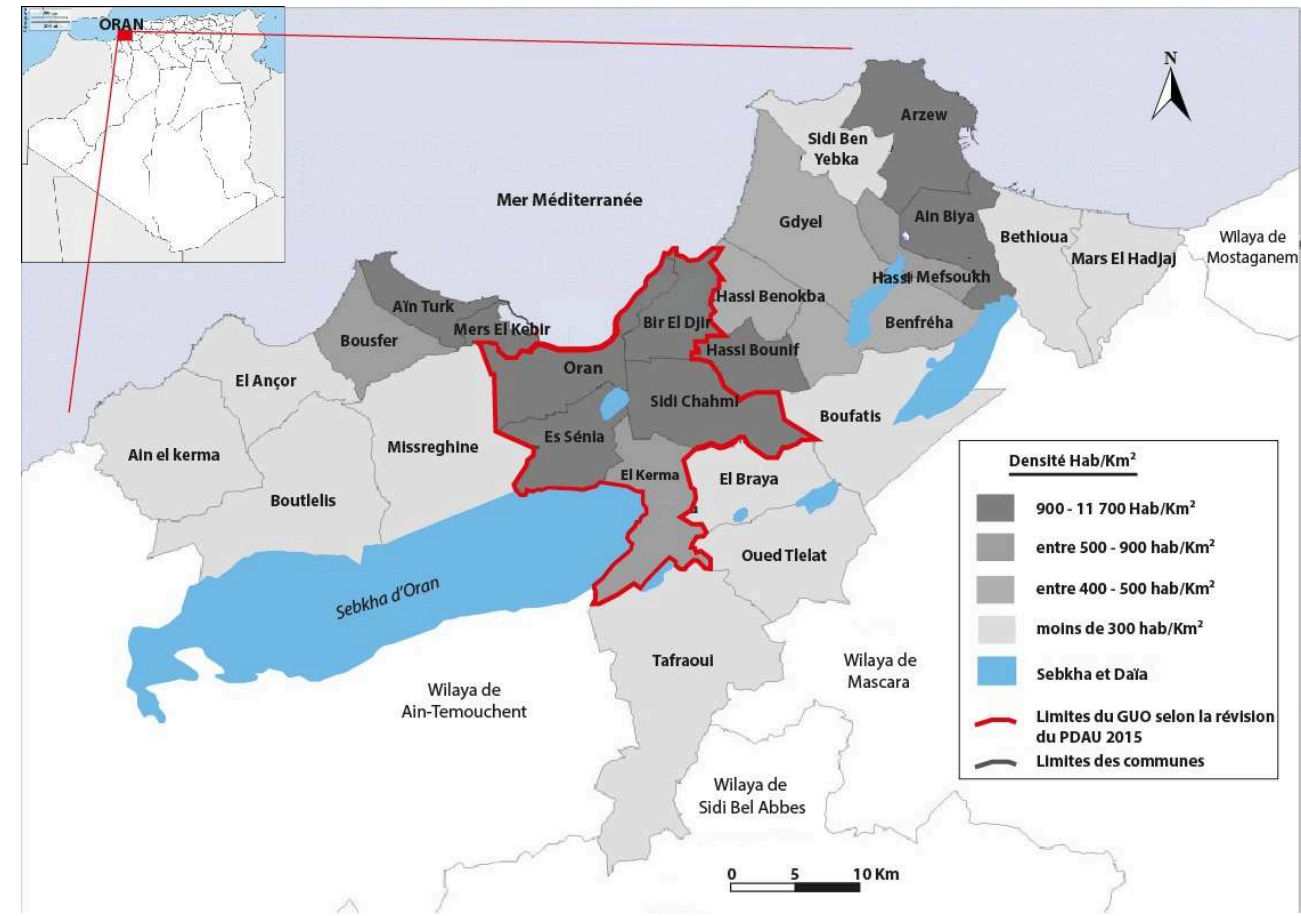

Source: Auteures ; 2019 sur la base du PDAU d'Oran (révision 2015) et de la carte de la répartition de la population d'Oran par commune (Agence Nationale d'Intermédiation et de Régulation Foncière ANIREF- 2018)

\section{L'espace agricole périurbain, une réserve foncière à disposition de la dynamique métropolitaine d'Oran}

L'espace périphérique agricole d'Oran correspondait au lendemain de l'indépendance à un riche terroir agricole de plaine ponctué par un semis de hameaux et de fermes; il renfermait de grandes propriétés agricoles. Le dynamisme démographique et socioéconomique d'Oran est à l'origine de l'étalement urbain et d'un processus 
d'urbanisation accéléré de ses espaces agricoles périphériques. Selon une étude basée sur un traitement des images satellitaires (Missoumi et al., 2019), la surface urbanisée de l'agglomération oranaise est passée de 6013 ha en 2008 à 7104 ha en 2017. Cet étalement s'est fait au détriment de terres agricoles fertiles ayant constitué durant des siècles un riche terroir fortement structuré par un semis de grandes fermes (fig. 2).

Figure 2 : L'occupation du sol à Oran en 1957.

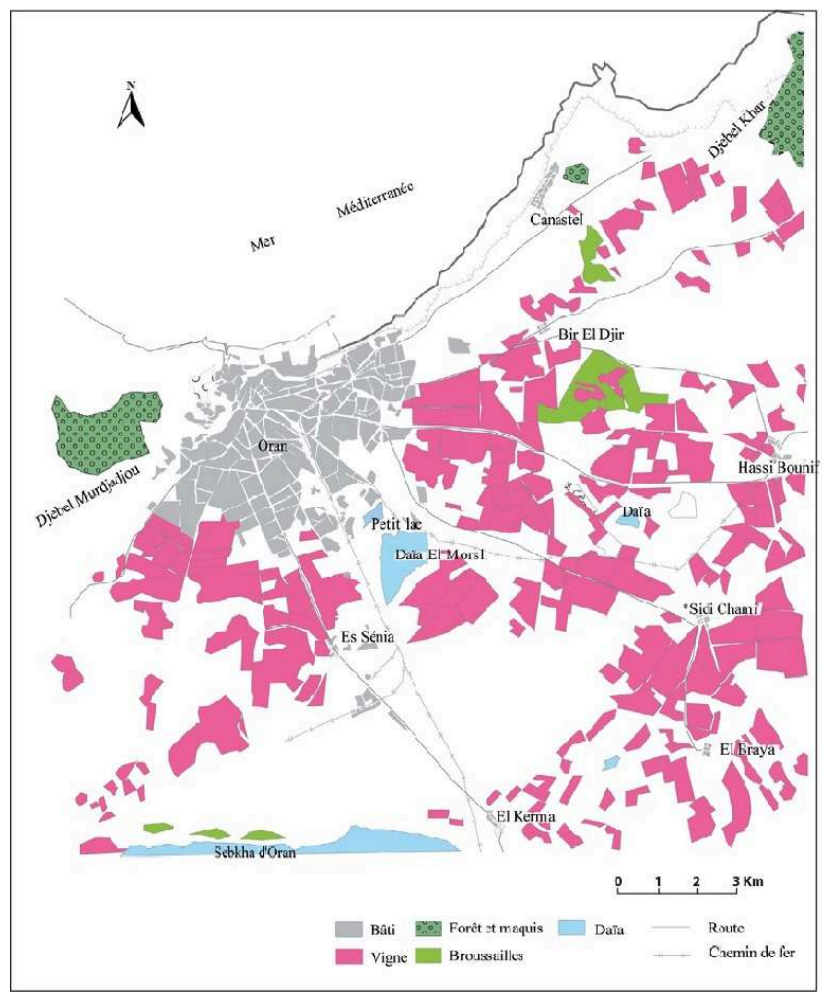

Source : Maachou H.M. et Otmane T., 2016

19 Après l'indépendance, l'espace agricole oranais subit, comme partout ailleurs en Algérie, l'application de la machine des réformes foncières. En 1983, les terres agricoles périurbaines de l'agglomération oranaise étaient organisées en 25 Domaines Agricoles Socialistes (DAS) de surface variant entre 400 ha et 500 ha (Maachou, 2008). À partir de 1987, l'éclatement des 25 DAS de l'agglomération donne naissance à 166 EAC et 1076 EAI couvrant respectivement 7380 ha et 5988 ha. C'est cet ancien territoire agricole renfermant des terres de bonne et très bonne qualité (les plaines d'Es Senia, Sidi Chahmi et Bir El Djir) qui a servi d'extension de l'agglomération oranaise.

Les meilleures terres agricoles des anciens domaines coloniaux (comme Si Redouane et Bahi Amar à Es Sénia) ont été utilisées d'assiette pour recevoir des programmes d'habitat. L'État, étant propriétaire majoritaire (tableau 1) et, en même temps, le principal planificateur, s'est donné l'autorisation de transformer le foncier agricole public en une ressource à la disposition du projet urbain, sans une réelle opposition.

Tableau 1. Groupement Urbain d'Oran (1998) : Répartition de la SAU par grands secteurs juridiques.

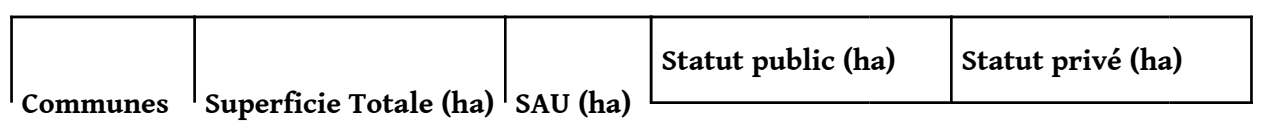




\begin{tabular}{|l|l|l|l|l|l|l|}
\cline { 4 - 7 } & & & Superficie (ha) & \% & Superficie (ha) & \% \\
\hline Oran & 6400 & 1010.34 & 719.09 & $\mathbf{7 1} \%$ & 291.25 & $\mathbf{2 9} \%$ \\
\hline Es Sénia & 5200 & 2054.24 & 1947.78 & $\mathbf{9 5} \%$ & 106.46 & $\mathbf{5} \%$ \\
\hline Bir El Djir & 4035 & 2617.02 & 2298.36 & $\mathbf{8 8} \%$ & 318.66 & $\mathbf{1 2} \%$ \\
\hline Sidi Chahmi & 9422 & 5019.44 & 4555.14 & $\mathbf{8 9} \%$ & 536.3 & $\mathbf{1 1} \%$ \\
\hline TOTAL & $\mathbf{2 5 0 5 7}$ & $\mathbf{1 0 7 7 3 , 0 4}$ & $\mathbf{9 5 2 0 . 3 7}$ & $\mathbf{8 8} \%$ & $\mathbf{1 2 5 2 . 6 7}$ & $\mathbf{1 2} \%$ \\
\hline
\end{tabular}

\section{Source : PDAU, 1998}

Dans le cadre du présent article, l'exploitation des plans parcellaires et des matrices cadastrales aurait eu une grande utilité dans l'analyse (spatio-juridique) de cette mutation des modes d'utilisation de l'espace agricole. Malheureusement, le Cadastre nous a, formellement, interdit d'exploiter la cartographie cadastrale pour une éventuelle reconstitution parcellaire du Groupement Urbain d'Oran, nous contraignant alors à se limiter à l'établissement d'une carte d'occupation du sol de notre zone d'étude à partir des images satellitaires datés de 2019 (fig. 3).

Figure 3. Occupation du sol de l'agglomération d'Oran (2019).

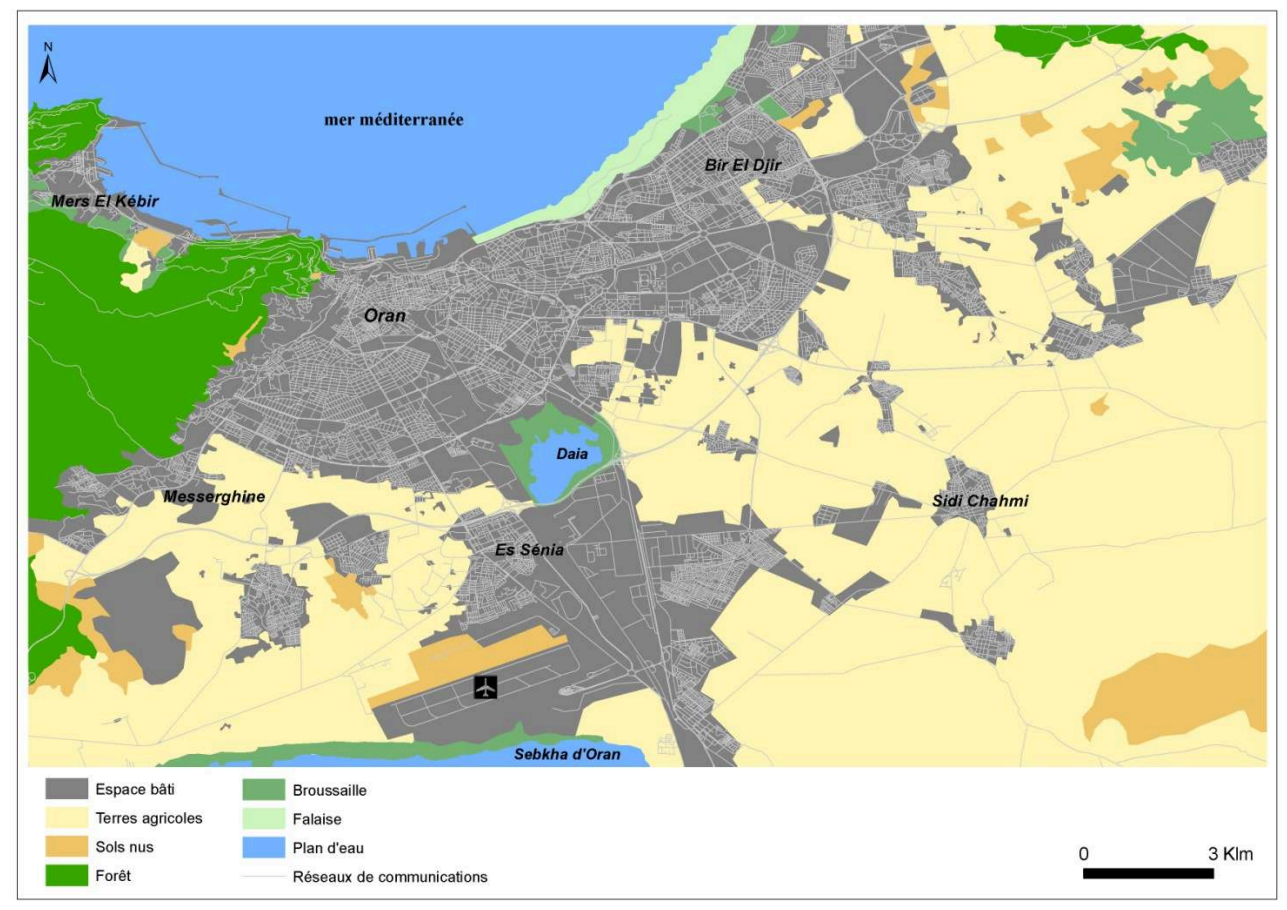

Source : Auteures, 2019

La préservation des terres agricoles semble être difficile et devient une problématique qui revient de façon récurrente et régulière. Selon une récente étude réalisée par Missoumi et al. (2019) sur la fragilisation de l'agriculture périurbaine dans l'agglomération d'Oran, la progression du bâti durant ces trente dernières années crée " un mitage en continu de l'espace agricole avec une moyenne annuelle de 42 hectares par an, 
soit l'équivalent de $1100 \mathrm{~m}^{2}$ de terres agricoles qui disparaissent quotidiennement au profit de l'implantation du tissu résidentiel et d'équipements " (Missoumi et al., 2019, p. 14), (tableau 2).

Tableau 2. Déperdition des terres agricoles face à la croissance urbaine dans l'agglomération Oranaise (1987-2017).

\begin{tabular}{|l|l|l|l|}
\hline $\begin{array}{l}\text { Période } \\
\text { d'étude }\end{array}$ & $\begin{array}{l}\text { Taux d'évolution des } \\
\text { surfaces urbanisées (\%) }\end{array}$ & $\begin{array}{l}\text { Surfaces agricoles cédées } \\
\text { à l'urbanisation } \\
\text { (ha) }\end{array}$ & $\begin{array}{l}\text { Taux annuel du } \\
\text { mitage agricole (ha/ } \\
\text { an) }\end{array}$ \\
\hline $\mathbf{1 9 8 7 - 1 9 9 8}$ & $+52,92$ & 548,24 & 50 \\
\hline $\mathbf{1 9 9 8 - 2 0 0 8}$ & $+16,50$ & 445,76 & 45 \\
\hline $\mathbf{2 0 0 8 - 2 0 1 7}$ & $+18,14$ & 265,69 & 30 \\
\hline
\end{tabular}

Source : Missoumi M.A. et al., 2019, « Jeux d'acteurs et fragilisation de l'agriculture périurbaine dans l'agglomération d'Oran (Algérie) », Études caribéennes [En ligne], pp. 43-44

Le détournement d'usage des terres agricoles, au profit de la réalisation d'équipements sociaux et économiques, constitue une infraction aux différents textes législatifs qui garantissent la protection du foncier agricole en interdisant sa convertibilité à d'autres usages que ceux qui lui ont été destinés au départ, à savoir : l'agriculture. Et pourtant, l'État accorde des exceptions à cette règle et promulgue deux décrets exécutifs en 2003 et en 2012 qui autorisent et facilitent la construction de logements et équipements publics sur des terres agricoles : le décret exécutif no 03-313 du 16 septembre 2003 qui fixe les conditions et les modalités de reprise des terres agricoles du domaine national incluses dans un secteur urbanisable, et le décret exécutif n ${ }^{\circ}$ 12-370 du 24 octobre 2012 portant déclassement de parcelles de terre affectées pour la réalisation de projets publics de développement (Missoumi et al., 2019).

\section{Quand Oran consume ses terres agricoles !}

En 1830, la ville d'Oran ne comptait que 4000 habitants. C'est durant la période coloniale que la ville, grâce à son port et son développement économique (basé sur la production viticole), devient la capitale de l'Ouest et une destination cotée pour l'immigration de la population de la métropole française. À l'indépendance et suite au départ des Européens, Oran hérite d'un parc immobilier important estimé à près de 40000 logements, ce qui fait d'elle une ville très privilégiée en matière d'habitat (Maachou, 2008). Le rythme de construction de logement s'est ralenti entre 1962 et 1975, alors qu'à cette période et afin de lancer rapidement la machine économique du pays le jeune État indépendant avait opté pour une politique d'industrialisation des grandes et des moyennes villes algériennes (Alger, Oran, Constantine, Annaba, Skikda). N'étant pas épargnée par cette nouvelle reconfiguration territoriale, Oran devint, une seconde fois, le territoire d'accueil de plusieurs vagues migratoires en provenance des petites villes et des villages voisins, augmentant ainsi les besoins en logement d'une population additionnelle toujours croissante. 
Au début de la décennie 1970, le tissu urbain d'Oran se limitait au niveau de la troisième couronne périphérique (le centre ancien espagnol et français et les faubourgs de la période coloniale) entouré par des vergers et des terrains agricoles (fig. 4).

Figure 4. Extension des espaces bâtis dans l'agglomération oranaise (1962-1997).

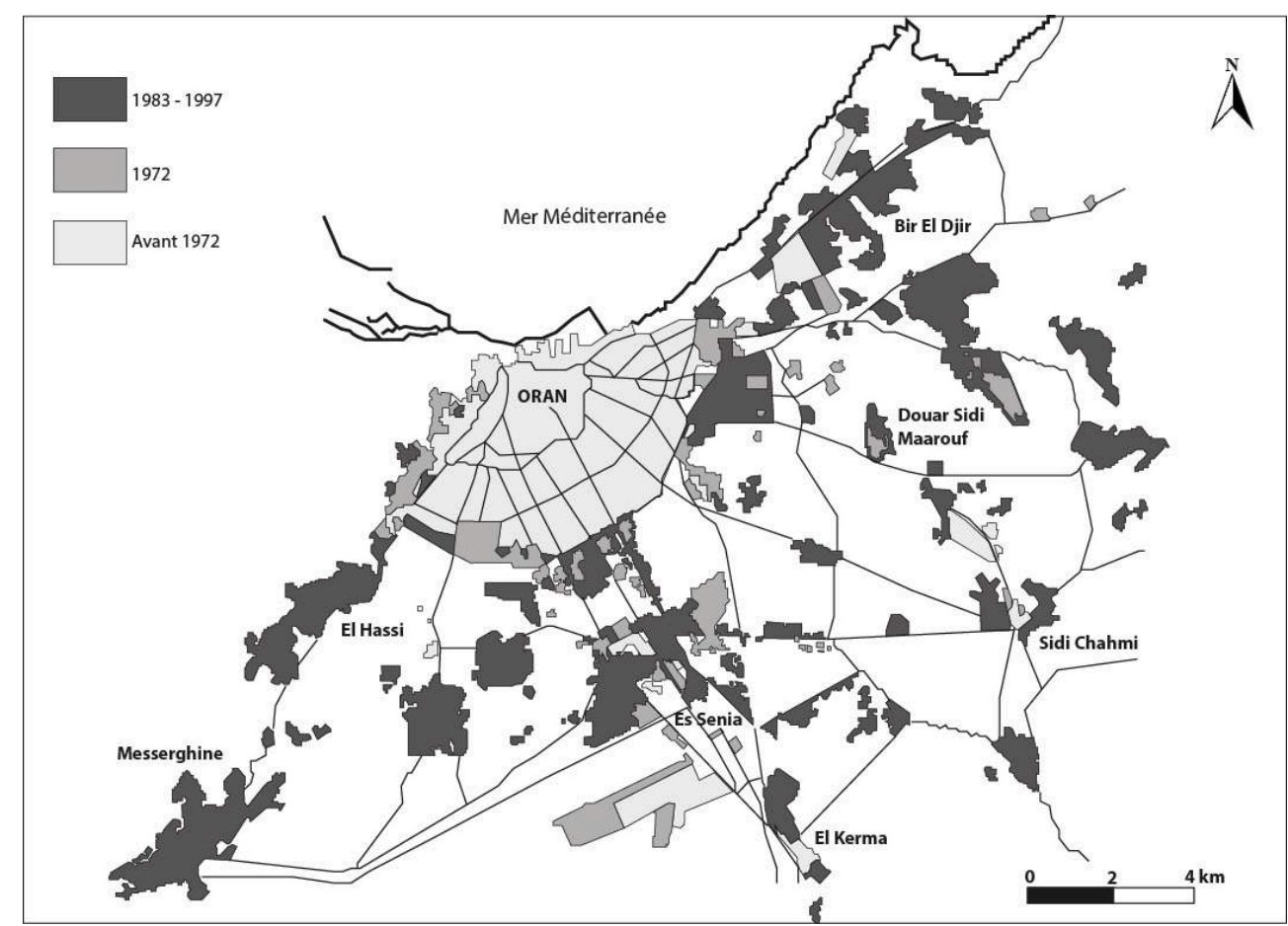

Source : Bendjelid et al., 2004

Devant une forte demande en logement, l'État se lance, à partir de 1975, dans la réalisation des projets d'urbanisation planifiée - les ZHUN (Zone d'Habitat Urbain Nouvelle) validés par le PUD (Plan Directeur d'Urbanisme) que les différents spécialistes de la question urbaine considèrent comme l'origine des principales extensions des périphéries des actuelles grandes villes algériennes. Dans l'agglomération Oranaise six ZHUN ont été réalisées sur une surface totale estimée à 1401 ha (tableau 3).

Tableau 3. Oran : les différentes ZHUN réalisées à partir de 1975.

\begin{tabular}{|l|l|l|}
\hline ZHUN & Superficie (ha) & Nombre de logements \\
\hline ZHUN Dar El Beïda & 150 & 1440 \\
\hline ZHUN Seddikia & 35 & 1240 \\
\hline ZHUN Maraval & 254 & 3350 \\
\hline ZHUN USTO & 150 & 1000 \\
\hline ZHUN Khemisti & 112 & 6000 \\
\hline ZHUN Fellaoucen & 700 & 1000 \\
\hline
\end{tabular}


\begin{tabular}{|l|l|l|}
\hline Ensemble ZHUN & 1401 & 14030 \\
\hline
\end{tabular}

Source : PDAU ORAN, 1997

À partir des années 1980, l'État s'est lancé dans la réalisation de programme d'habitat individuel dans le cadre des coopératives immobilières et des lotissements. Le Plan d'Urbanisme Directeur (PUD), élaboré et approuvé en 1977, avait indiqué les grandes lignes du développement urbain et de l'extension spatiale des zones Est et Nord-est de l'agglomération oranaise. Le choix des terrains de l'extension en question aurait été contesté par les services de l'agriculture, qui révoqueraient un développement urbain au détriment des terrains agricoles considérés, généralement, de bonne qualité (Direction des Services Agricoles de la wilaya d'Oran). Cela n'a pas empêché la réalisation des grands projets d'habitat et d'équipements, dont l'Université des Sciences et de la Technologie d'Oran (USTO) sur 89 ha, conçue par l'architecte japonais Kenzo Tange et inaugurée en septembre 1986. L'emplacement de l'Université a généré une "impulsion tout à fait considérable aux processus de périurbanisation qui ont reconfiguré en profondeur l'agglomération oranaise " (Kadri, Madani, 2015, p. 23). En cette période "la superficie urbanisée va croitre de 1254 ha dont les deux tiers se situent dans les espaces périphériques» (Kadri, Madani, 2015, p. 23). En s'inscrivant dans une logique d'urgence et de rattrapage, l'État s'est investi dans la concrétisation d'immenses programmes de construction non inscrits dans le document d'urbanisme, le PUD, dont la mission était la planification spatiale du développement urbain de l'agglomération oranaise pour la période 1977-1987. Cela a été une autre circonstance pour que des terres agricoles servent d'assiette d'urbanisation.

Après 1987, date d'expiration du PUD, l'agglomération oranaise a continué de croitre de manière considérable, mais en l'absence d'instrument d'urbanisme. Pendant quatre ans (de 1987 à 1990) on assiste à une urbanisation sans plan ni orientations urbanistiques.

Durant la décennie 1990 (que l'on qualifie de décennie noire) marquée par un important exode rural, la ville-mère étant saturée, l'urbanisation d'Oran s'est reportée à l'est de l'agglomération sur les communes périphériques (fig. 5), matérialisée par le grignotage de vastes surfaces présentant le moins de contraintes au gré des disponibilités foncières et entrânant de fait son débordement (Benkada, 2001).

Figure 5. Commune de Bir-El-Djir : constructions d'habitats individuels et collectifs aux limites des terres agricoles.
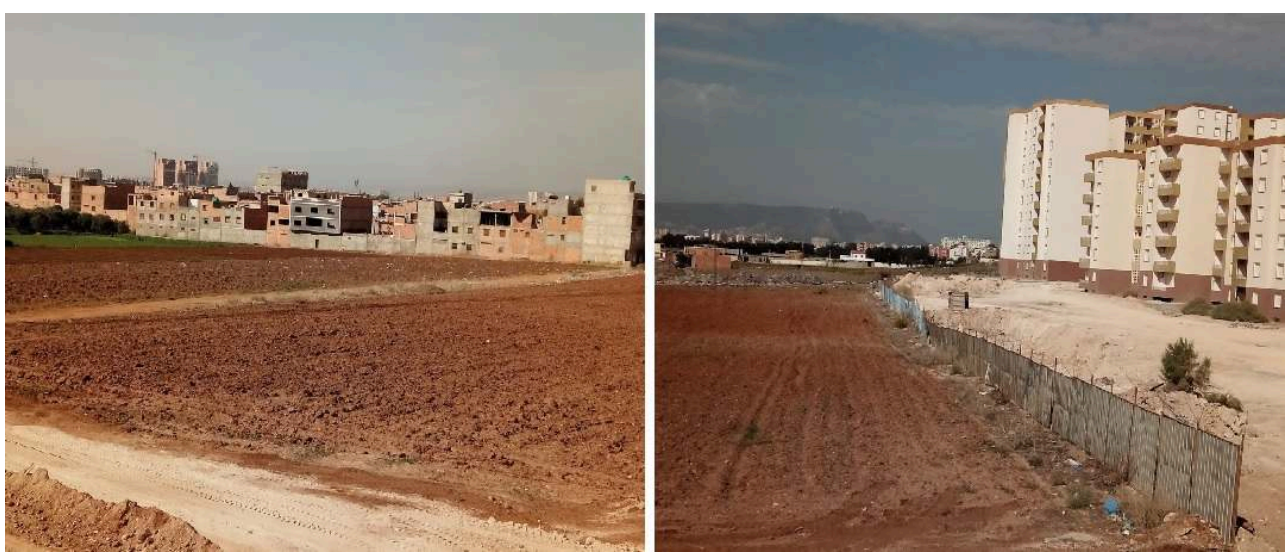

Source : Auteures (septembre 2019) 
Au regard des conditions d'urbanisation, bien des questions surgissent et notamment pourquoi l'État a-t-il amputé l'instrument d'urbanisme de sa mission essentielle et pourquoi a-t-il enfreint ses propres lois? L'instrument d'urbanisme ne serait-il qu'un simple alibi et la planification spatiale de l'agglomération oranaise ne serait-elle pas en panne? (Guerroudj, 2004).

\section{PDAU et POS, des instruments d'urbanisme contrariant la protection des terres agricoles périurbaines}

En 1990, l'Algérie remet en cause les précédents dogmes de la politique d'aménagement du territoire et de la planification urbaine. La loi no $90-29 \mathrm{du} 1^{\text {er }}$ décembre 1990 relative à l'aménagement et l'urbanisme instaure un nouveau cadre juridique de la planification spatiale en termes de règles fonctionnelles et de normes d'occupation du sol. Deux instruments d'urbanisme et d'aménagement sont mis en place à savoir: le Plan Directeur d'Aménagement et d'Urbanisme (PDAU) à l'échelle des agglomérations et le Plan d'Occupation du Sol (POS), en tant qu'instrument de détail.

\section{Le Plan Directeur d'Aménagement et d'Urbanisme (PDAU), un instrument a priori en faveur d'une protection des terres agricoles}

Le PDAU, élaboré dans les années 1990, avait eu pour première fonction la délimitation du territoire correspondant au Groupement Urbain d'Oran. Ce dernier occupait une position centrale dans sa wilaya (Oran) ; il réunissait quatre communes (Oran, Es Senia, Bir el-Djir et Sidi Chahmi) et s'étendait sur une surface de 25057 ha. La surface urbanisée occupait plus de 8800 ha soit $35 \%$ de la superficie totale du groupement. Le reste de la superficie, soit $65 \%$, se composait de terres agricoles, de forêts, de terres incultes, etc. (PDAU, 1997). Le PDAU préconisait une planification spatiale basée sur la méthode du zoning : il avait réparti les espaces d'urbanisation en secteurs. Ces derniers étaient des fractions continues du territoire communal et se distinguaient chacune par l'une des quatre vocations fixées par la loi 90-29 (article 19) : secteur urbanisé (SU), secteur à urbaniser (SAU), secteur d'urbanisation future (SUF) et secteur non urbanisable (SNU) (tableau 4).

Tableau 4. Oran 1997 : répartition des secteurs d'urbanisation selon le PDAU.

\begin{tabular}{|l|l|l|l|l|}
\hline $\begin{array}{l}\text { Catégorie } \\
\text { de vocation }\end{array}$ & Types de terrains concernés & $\begin{array}{l}\text { Superficie } \\
\text { (ha) }\end{array}$ & $\begin{array}{l}\text { Secteur d'espace } \\
\text { concerné }\end{array}$ & $\begin{array}{l}\text { Nbre } \\
\text { de } \\
\text { POS }\end{array}$ \\
\hline SU & $\begin{array}{l}\text { Ensemble des terrains occupés par les } \\
\text { constructions agglomérées }\end{array}$ & 8860 & Oran & 48 \\
\hline SAU & $\begin{array}{l}\text { Terrains destinés à être urbanisés dans } \\
\text { une perspective d'extension à court et à } \\
\text { moyen terme }\end{array}$ & 704 & $\begin{array}{l}\text { Le Groupement d'Oran } \\
\text { Urbain }\end{array}$ & 5 \\
\hline
\end{tabular}




\begin{tabular}{|l|l|l|l|l|}
\hline SUF & $\begin{array}{l}\text { Terrains destinés à être urbanisés à un } \\
\text { horizon de } 20 \text { ans }\end{array}$ & 960 & $\begin{array}{l}\text { Terrains de la zone } \\
\text { Est du Groupement } \\
\text { Urbain d'Oran }\end{array}$ & 7 \\
\hline SNU & $\begin{array}{l}\text { Les espaces où les droits à construire } \\
\text { étaient considérés comme nuls ou limités } \\
\text { (loi 90-29, art.23) : les différents terrains } \\
\text { agricoles, la frange littorale et les forêts. }\end{array}$ & 14533 & Tout Oran & 6 \\
\hline
\end{tabular}

Source : PDAU d'Oran, 1997

Le Plan d'Occupation du Sol (POS) : entre affectation et réglementation des droits d'usage des sols dans les zones périurbaines

33 Le POS est un instrument réglementaire qui fixe les droits de construction. D'après l'article 31 de la loi 90-29, le POS fixe de façon détaillée les droits d'usage du sol et de construction, mais toujours dans le cadre des dispositions énoncées par le PDAU. Cet instrument se décline en deux types : le premier « d'aménagement ou d'extension », qui a pour objectif de cerner et de réglementer les nouveaux terrains urbanisables, privilégie les terrains vierges, en situation de périphérie; le second appelé «POS de restructuration ", intervient à l'intérieur de l'agglomération. Dans le cas du PDAU d'Oran, 66 POS ont émergé et sont toujours en vigueur actuellement (Kadri , Madani, 2015).

34 Selon les différents travaux de recherches consultés (articles et thèses de doctorat), l'ampleur de l'étalement de la ville d'Oran a été telle que les secteurs à urbaniser (SAU) ont été rapidement consommés, que les secteurs d'urbanisation future (SUF) ont déjà fait l'objet d'urbanisation et que les terres agricoles ont été urbanisées malgré le corpus législatif et réglementaire en faveur de leur préservation.

Ces faits observés sont indicateurs des faiblesses des instruments d'aménagement et d'urbanisme face la dynamique démographique, sociale et économique puisqu'ils deviennent, souvent au moment de leur application, incohérents et caducs et qu'ils bravent l'opposabilité au tiers et ne résultent pas de la concertation et de la consultation publique (de telles exigences des instruments d'aménagement et d'urbanisme ne deviennent qu'une question de forme, d'alibi).

\section{L'urbanisation des terres agricoles de la périphérie oranaise: par quelles stratégies d'acteurs?}

Dans ce dernier volet de l'article, il est question d'établir un état de fait pratique et concret sur les modalités de consommation des terres agricoles périurbaines d'Oran. Cet objectif de terrain reflète, en même temps, la démonstration de toute une réflexion théorique qui met en exergue quelques notions essentielles à la problématique de l'urbanisation des terres agricoles, à savoir les notions d'acteurs, de stratégies et d'actions.

37 Pour Henri Lefebvre (2005), l'espace réel est celui de la pratique sociale, autrement dit l'espace est en perpétuelle construction. La ville étant une production sociale, elle est le 
produit de tout un processus de transformations spatiales basées, essentiellement, sur le transfert du foncier périurbain (agricole ou autre) en tant que ressource pour le développement du projet urbain. L'espace périurbain serait, de ce fait, l'objet de confrontations entre acteurs (individus, collectivité territoriale, associations, etc.) aux stratégies divergentes (développement de la ville ou préservation des terres agricoles). Dans la majorité des cas, le caractère agricole du foncier périurbain apporte à la question foncière la dimension de ressource. La réflexion en termes de stratégies nous oblige, donc, à comprendre la rationalité des acteurs dans leurs actions et leur processus organisationnel. "Toute étude sur la production foncière et immobilière implique une identification des acteurs et des mécanismes en jeu» (Ameur, 1993, p. 16). Dans le présent article, il est entendu par production foncière le processus dont l'aboutissement est la mise à la disposition de l'État constructeur ou des promoteurs immobiliers de lots de terrain à bâtir.

La multiplicité des acteurs reflète, nécessairement, une diversité de la production foncière, en faveur des demandeurs potentiels des lots à bâtir. Cet état de réflexion nous amène au concept de filière/animateur de Durand-Lasserve et Tribillion (1983) qui appréhendent dans leurs travaux trois types de filières dans la production du foncier à bâtir : la filière capitaliste/le promoteur, la filière publique/l'État et la filière petite marchande-populaire/petits producteurs-habitants. Pour notre part, nous nous intéressons à la filière publique/l'État. Ce choix est justifié par le fait que l'État algérien reste le propriétaire majoritaire des meilleures terres agricoles du pays et il est considéré, en même temps, comme le plus grand consommateur de lots de terrains agricoles. Cette consommation excessive du foncier périurbain au profit des villes est étiquetée par les spécialistes comme une mauvaise manœuvre utilisée par l'État pour combler et réguler les déficiences d'ordre économique (zones industrielles), social (logements, services, etc.) et politique (élus qui utilisent le foncier pour des fins électorales, etc.). Il serait donc intéressant de mettre en lumière ce processus d'urbanisation sur les terres agricoles du périurbain de l'agglomération oranaise.

À Oran, cet espace de transition entre le rural et la ville a connu de profondes mutations depuis une trentaine d'années. "Ni la constitution des réserves foncières au profit des communes (Loi de 1974), ni l'instruction présidentielle $n^{\circ} 13$ d'avril 1987, ni la nouvelle loi d'orientation foncière (Loi 1990) n'ont réussi à préserver les terrains agricoles de l'invasion urbaine» (Trache, 2010). L'enquête de terrain réalisée auprès de quelques acteurs de l'aménagement du territoire de l'agglomération oranaise (Direction de l'Urbanisme et de la Construction de la wilaya d'Oran, Direction des Services Agricoles de la wilaya d'Oran, les collectivités territoriales et certains experts géographes qui ont participé à la réalisation des PDAU et des POS) a révélé que l'urbanisation et/ou le détournement du foncier agricole s'opèrent en deux phases successives que nous décrivons ci-dessous.

\section{Une première phase : récupération/déclassement des terres agricoles étatiques par les instruments d'urbanisme}

En Algérie, l'augmentation des besoins en foncier urbanisable pour la réalisation de logements et d'infrastructures publiques a engendré une pression grandissante sur le foncier agricole. En fait, les pouvoirs publics se sont retrouvés devant un dilemme: celui de préserver les terres agricoles pour assurer la sécurité alimentaire des futures 
générations et celui de mener à bon port les programmes de développement territorial, notamment ceux d'utilité publique.

Jusqu'à 2010, la transformation du foncier agricole en foncier urbanisable s'effectue selon un processus administratif basé sur le principe de l'utilité publique. L'État récupère les parcelles voulues, souvent appartenant au domaine public ou privé de l'État (EAC ou EAI) avec l'approbation d'une commission de wilaya dans laquelle le représentant de la Direction des Services Agricoles participe mais n'exerce aucun ou peu de pouvoir pour freiner ce processus. Dès lors, plusieurs hectares de terres à haute valeur agricole, dans le périurbain des grandes et des moyennes villes algériennes, ont été intégrés au projet du développement des villes et des infrastructures publiques. À cette période, l'urbanisation de la ville d'Oran a été la résultante d'une consommation démesurée et progressive des meilleures terres agricoles, une situation paradoxale dans la mesure où cette même urbanisation est produite et encadrée par des documents d'aménagement et d'urbanisme, en l'occurrence le PDAU et les POS (tableau 5).

Tableau 5. Oran (1997) : superficie des terres agricoles récupérée par le PDAU au profit de l'urbanisation.

\begin{tabular}{|l|l|l|l|}
\hline Communes & $\begin{array}{l}\text { Secteur à } \\
\text { urbaniser (ha) }\end{array}$ & $\begin{array}{l}\text { Urbanisation } \\
\text { future (ha) }\end{array}$ & $\begin{array}{l}\text { Surface agricole totale affectée } \\
\text { à l'urbanisation (ha) }\end{array}$ \\
\hline $\begin{array}{l}\text { Le Groupement Urbain } \\
\text { d'Oran }\end{array}$ & 733.16 & 980.63 & $1713.7^{*}$ \\
\hline $\begin{array}{l}\text { Les 22 communes de la } \\
\text { wilaya d'Oran }\end{array}$ & 570.44 & 579.98 & $1150.42^{*}$ \\
\hline Total Wilaya & 1303.6 & 1568.61 & 2864.25 \\
\hline
\end{tabular}

*Sur la surface totale des terres agricoles affectées à l'urbanisation dans le groupement d'Oran, 505 ha sont repris par un simple arrêté de wilaya ; pour les 22 autres communes, on note 193 ha repris par arrêté de wilaya.

Source : Direction des Services Agricoles

Selon les informations établies dans le tableau 5, près de 3000 hectares de terres agricoles sont affectés à l'urbanisation actuelle ou future à l'échelle du territoire de la wilaya d'Oran, dont $60 \%$ sont affecté à la seule extension du Groupement Urbain d'Oran (GUO).

Dans cette même période, une nouvelle opération de récupération des terres agricoles est lancée par le pouvoir central. Il s'agit du décret exécutif $n^{\circ}$ 03-313 du 16 septembre 2003 fixant les conditions et les modalités de reprise des terres agricoles du domaine national intégrées dans un secteur urbanisable. Tout wali (Préfet) a l'autorité de prononcer, par arrêté, la reprise d'un lot de terrain agricole au profit de l'État et d'indemniser les propriétaires ou les attributaires des EAC et EAI (lorsqu'il s'agit du domaine privé de l'État). Dans le cas de la wilaya d'Oran, 698 ha $^{11}$ de terres agricoles ont été repris par un simple arrêté de wilaya (décision préfectorale) et versés dans les zones à urbaniser délimitée par le PDAU. Il est tout à fait clair que la résistance à l'urbanisation de certaines parcelles agricoles n'est qu'une manière d'augmenter la spéculation foncière dans la zone périurbaine. Certains propriétaires privés dont les terres 
agricoles se situent à l'intérieur du périmètre urbanisable ont vu la valeur de leur bien foncier augmenter à des prix exagérés. Ce détournement 'légal' de la fonction agricole du foncier périurbain n'est que le résultat d'un processus enclenché par les services publics, en commençant par le PDAU qui applique son zonage sur des espaces agricoles en les intégrant dans les zones urbanisables. Le pouvoir exécutif (la wilaya) apporte sa touche législative en légalisant ce transfert de foncier agricole par l'application de lois et décrets basés sur le principe de l'utilité publique.

Il est fort de constater que le PDAU devient un instrument d'urbanisme qui sert à produire du sol urbanisable pour les extensions futures (Kettaf, 2013). Ce document d'urbanisme tend à s'éloigner, petit à petit, des orientations officielles qui lui sont assignées, c'est-à-dire : orienter l'extension de la ville, reconquérir les tissus urbains existants et préserver les terres agricoles. Ce mode opératoire est l'expression affirmée du rapport de force qui existe entre les services agricoles et le pouvoir exécutif, car de telles pratiques pourraient étonner quand on sait que lors de l'élaboration des documents d'Urbanisme (PDAU, POS), les services agricoles sont obligatoirement consultés.

En 2011, suite à la création de l'Office National des Terres Agricoles et sous la pression du mécontentement social en rapport aux droits d'accès au logement, le gouvernement algérien procède à la promulgation de textes réglementaires relatifs au déclassement $\mathrm{du}$ foncier agricole à moyen et à faible rendements ${ }^{12}$. Les parcelles récupérées sont transformées en réserves foncières de la politique urbaine. Au niveau national cette question de déclassement de terrains agricoles a suscité beaucoup de débats. Dans un entretien accordé à un journal national (le Soir d'Algérie) en décembre 2018, le directeur central de l'Organisation Foncière et la Protection des Patrimoines au Ministère de l'Agriculture et du Développement Rural affirme que 40000 ha ont été déclassés à travers le territoire national. Ce constat pousse, aujourd'hui, le pouvoir central à revoir sa politique de gestion du foncier agricole. "Aujourd'hui, il faut revenir à l'orthodoxie. Il faut revenir aux instruments d'urbanisme et à la législation. Le déclassement des terres agricoles devient un impératif extrême. Encore, faut-il que ces terres soient de très faibles valeurs agricoles » (Hamdani, 2018).

Selon nos entretiens réalisés auprès des acteurs publics de l'agglomération oranaise (Direction de l'Urbanisme et de la Construction de la wilaya d'Oran, Direction des Services Agricoles de la wilaya d'Oran, Service d'Urbanisme de l'APC de Bir El Djir), le déclassement des terres agricoles périurbaines au profit de l'extension de la ville se fait avec l'approbation de la direction des services agricoles de la wilaya. À titre d'exemple, plus de 600 ha de terres agricoles ont été déclassés dans la commune de Bethioua, un territoire de la wilaya d'Oran, au profit de la nouvelle zone industrielle: plusieurs entreprises industrielles y sont implantées à l'image de l'importante usine de la société turque Tosyali.

Mais comment peut-on déclasser un terrain, considéré depuis plusieurs générations comme à haute valeur agricole, au rang de terrain inculte ou à faible potentialité ? Notre interrogation est restée posée devant une administration muette fuyant de répondre en affirmant que toutes les parcelles agricoles déclassées sont justifiées sans donner de détails sur la procédure en question. "Notre consultation à l'égard de l'utilité agricole ou non de certains terrains reste de l'ordre de la formalité administrative. Lorsqu'il s'agit du foncier public et même du privé de l'État, notre avis ne pèse pas assez sur la balance agriculture/développement urbain... les terrains en question sont le bien de l'État et il est libre 
d'en faire ce qu'il veut...toutes les stratégies employées par l'État sont dans un cadre législatif légal et la DSA ne peut rien y faire » (ingénieur agronome, DSA d'Oran).

\section{Une deuxième phase : les POS et la réaffectation illicite de l'usage des îlots planifiés}

Lorsque le PDAU met en place son zonage, essentiellement les zones à urbaniser et les zones à urbanisation future, le périmètre est divisé en POS dans le but d'organiser l'usage des espaces produits. C'est à ce niveau de l'affectation des îlots du POS que s'effectuent les différents détournements et pratiques illicites sur le foncier à urbaniser (autrefois agricole). Ce foncier, qui est détourné une première fois par son intégration aux zones à urbaniser, sera détourné une deuxième fois par son affectation à des usages autres que ceux prévus par les instruments d'urbanisme.

En guise d'illustration, nous avons choisi d'apporter un éclairage sur les pratiques de détournement observées au niveau de la commune de Bir El Djir, commune qui a suscité beaucoup de débats et a fait l'objet de plusieurs scandales fonciers ${ }^{13}$. Cette commune qui occupe la partie nord-est du Groupement Urbain d'Oran et qui s'étend du nord au sud, sur une distance de $5 \mathrm{~km}$ (Cf. fig. 1), est composée d'une Agglomération Chef-Lieu (ACL) Bir El Djir et deux Agglomérations Secondaires(AS) : Sidi el Bachir Bendaoud et Belgaid. Elle a été choisie pour supporter l'extension Est du Groupement Urbain d'Oran (fig. 5). Sur une surface communale totale de 4035 ha (surface totale de Bir El Djir), 2617 ha sont classées SAU (Surface Agricole Utile) dont 900 ha sont intégrés dans les Secteurs à Urbanisation Future (SUF). En matière d'occupation du sol, les terres agricoles de Bir El Djir sont dominées, essentiellement, par des cultures annuelles (la céréaliculture et maraîchage) et par des cultures permanentes (des vergers d'agrumes), les terres non utilisées par l'agriculture sont des parcours ou des forêts.

Parmi les objectifs du PDAU figure la détermination des périmètres d'intervention des POS. À cet effet, le PDAU a procédé à un découpage du secteur de Bir el Djir en zones homogènes identifiées à partir d'une carte d'occupation du sol. Le territoire communal de Bir el Djir a été divisé en 18 zones, ce qui correspondait à 18 plans d'occupation de sol (POS) dont quatre POS approuvés, 12 POS en cours d'élaboration et deux POS non encore élaborés. 
Figure 6. Évolutions des secteurs d'urbanisation dans la commune de Bir El Djir (1997-2015).

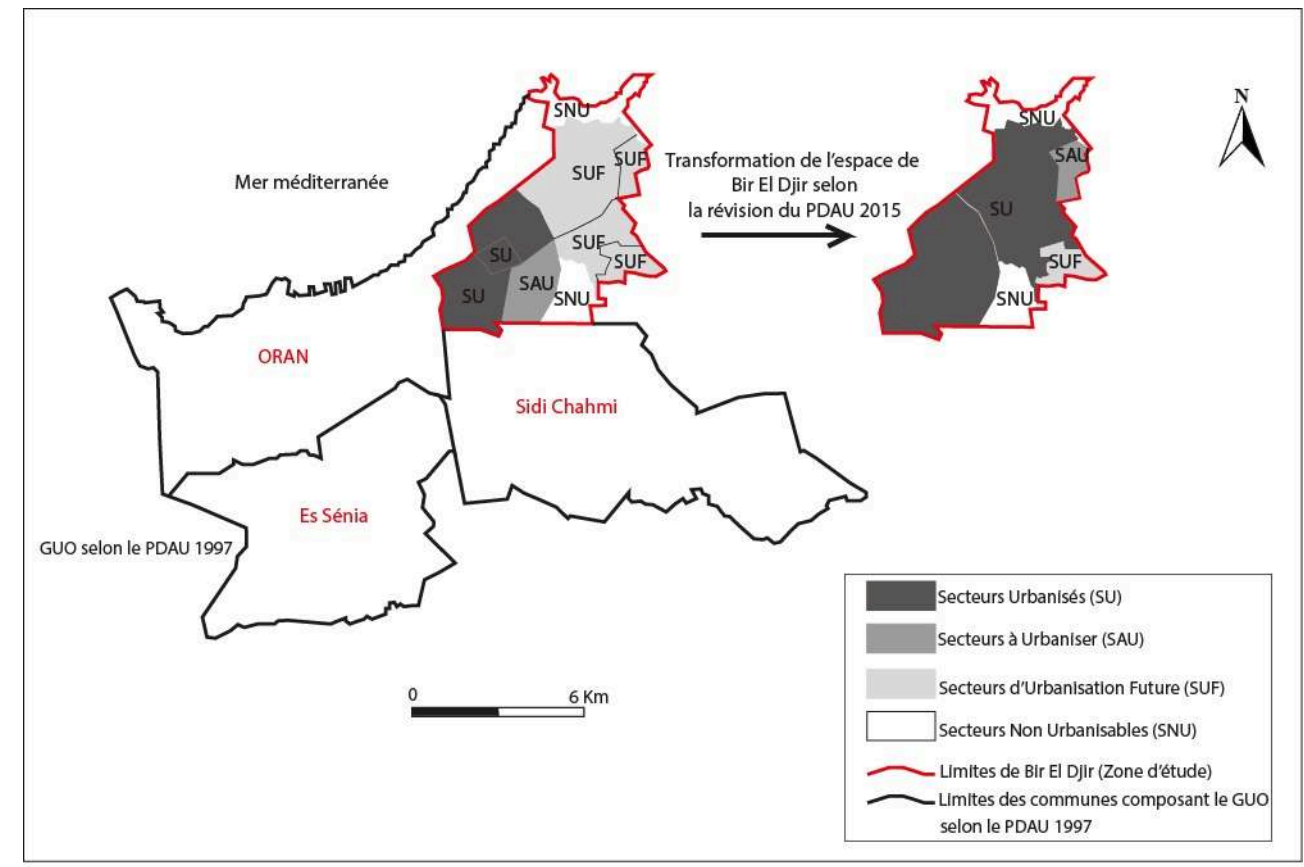

Source : Reconstitution par l'auteures sur la base de la cartographie du PDAU d'Oran 1997 et la révision du PDAU en 2015.

51 Sur le terrain, il est fort de constater la consommation presque totale du foncier de Bir El Djir par l'urbanisation, malgré la non approbation de certains POS et parfois, même, le non achèvement de leurs études. C'est le cas du territoire de trois POS $(21,25,49)$ et la zone des SUF (SUF5, SUF4, SUF3) de Bir El Djir qui ont été entièrement consommés avant même l'élaboration et l'approbation des documents d'urbanisme (POS) (fig. 7). Cette situation est révélatrice d'un dysfonctionnement administratif et d'une absence de gouvernance foncière, autorisant ainsi toutes les formes de détournements et de trafics fonciers. 
Figure 7. Commune de Bir-El-Djir (2003) : état d'avancement des POS planifiés par le PDAU en 1997.

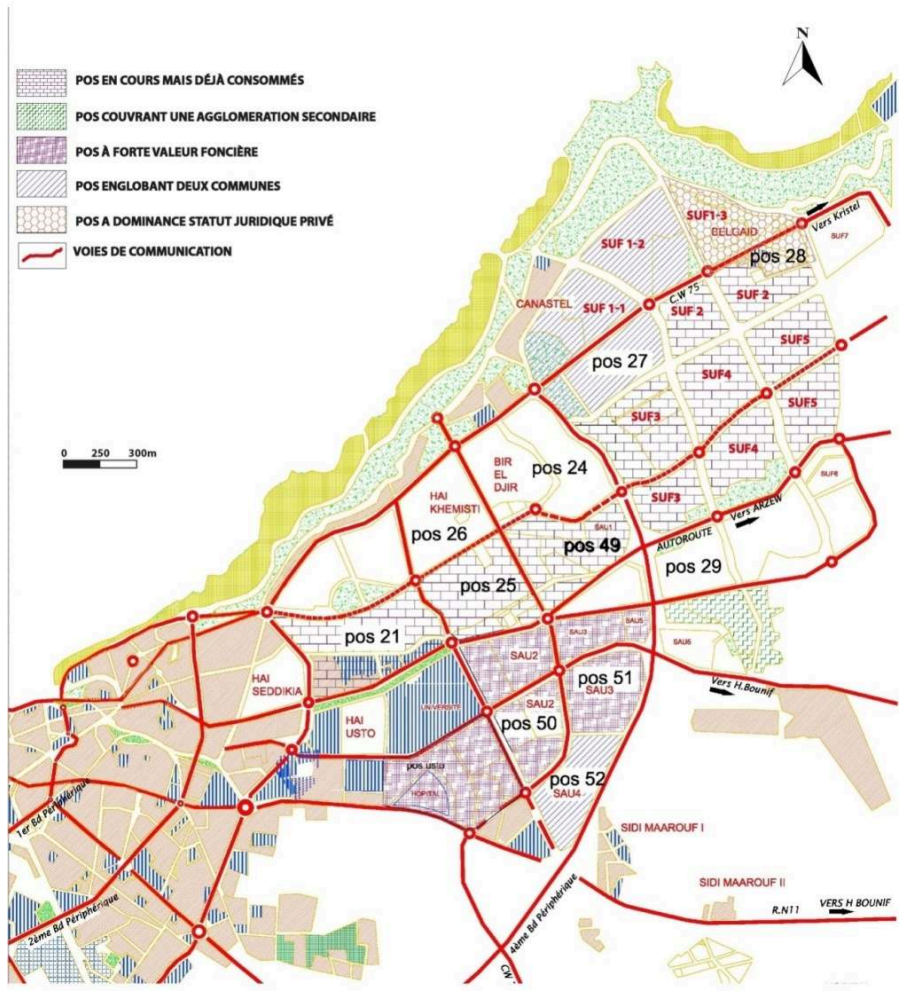

Source : Zouani, 2016, p. 70

Dans le discours recueilli auprès des acteurs de l'urbanisme, le caractère d'urgence représente la raison des différentes actions d'urbanisation des espaces périurbains de la ville d'Oran. L'explosion démographique de la métropole oranaise exerce une pression insupportable sur le pouvoir exécutif. "C'est dans un climat d'urgence que les pouvoirs centraux et locaux se sont efforcés à faire face à des demandes sociales toujours en croissance et plus particulièrement en matière de logement » (Zouani, 2016).

Souvent, la réalisation des programmes de logements ou d'équipements publics se fait sur la base d'un simple accord établi dans un procès-verbal de la commission de choix de terrain à l'échelle de la daïra ou celle de la wilaya. Dans la commune de Bir El Djir, nous pouvons citer deux exemples de réaffectation des sols :

- Le stade olympique d'Oran (fig. 8), réalisé au SUF3 de la commune de Bir El Djir, a été initialement prévu au SUF 1 par le PDAU 1998. Selon le témoignage d'un ancien expert géographe, le choix final du terrain du stade olympique a été imposé par l'exécutif central à Alger. «La vision des personnes proches du pouvoir central est complètement décalée par rapport à notre vision, nous, les praticiens de l'aménagement du territoire. Dans la phase étude du PDAU 1998, nous avons prévu une ceinture verte au-delà du périphérique 4. C'était une manière de préserver l'agriculture périurbaine à Oran. Or, à Alger les objectifs étaient les Jeux méditerranéens et l'image politico-touristique de la métropole oranaise. Ils ont donc imposé la construction du stade olympique aux portes de l'agglomération et sur des terres agricoles » (ancien expert géographe d'un bureau d'étude privé).

- Cette décision centralisée montre, encore une fois, l'écart qui se trouve entre «la planification à la verticale » et la réalité des territoires. Il n'est pas exclu que certaines de ces 
pratiques foncières relatives à la réaffectation d'usage des îlots planifiés s'opèrent dans un contexte de clientélisme

Figure 8. Stade olympique d'Oran.

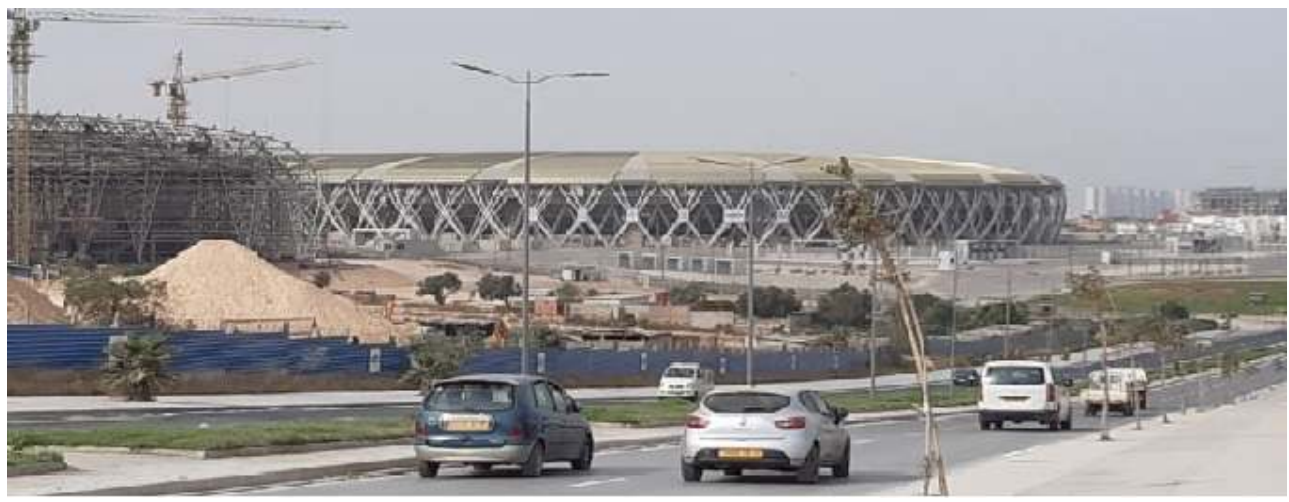

Source : Auteures (2020)

- Dans la partie sud du POS 51 de Bir El Djir (fig. 9), deux parcelles qui ont été initialement affectées par le POS 51 (1998) à la réalisation respectivement d'un lycée technique et d'un espace de loisir (espace vert), ont servi d'assiette à la promotion immobilière: des résidences de haut standing, souvent inaccessibles aux couches moyennes de la société, sont en construction (fig. 9) sans la moindre contestation des services concernés (l'APC de Bir El Djir, la commission des choix de terrains, etc.).

« Le POS 51 est, majoritairement, de statut juridique domanial. Il s'étale sur une surface de plus de 25 ha et constitue un des POS les plus contesté à Bir El Djir... sa partie sud a subi plusieurs réaffectations d'usage d'îlots déjà planifiés et approuvés, mais cette pratique est très fréquente " (Z.R ingénieur aménagiste au Service de l'Urbanisme et de l'Habitat à la mairie de Bir El Djir). La technique consiste à attribuer une nature d'occupation de sol "floue“ aux îlots du POS. Pour notre cas d'étude, plus de 20 parcelles sont affectées à ce qu'on appelle équipements d'accompagnement ou d'investissement (école, crèche, hôtel, centre commercial, centre d'affaire, etc.). «La liberté de cette réaffectation d'usage des îlots planifiés s'explique par deux éléments majeurs : le premier est le statut domanial des propriétés foncières composant les POS et le second est l'urgence qui caractérise la plupart des interventions et des demandes officielles " (Z.R ingénieur aménagiste au Service de l'Urbanisme et de l'Habitat à la mairie de Bir El Djir). 
Figure 9. Commune de Bir El Djir : réaffectation d'usage d'un îlot dans le POS 51.

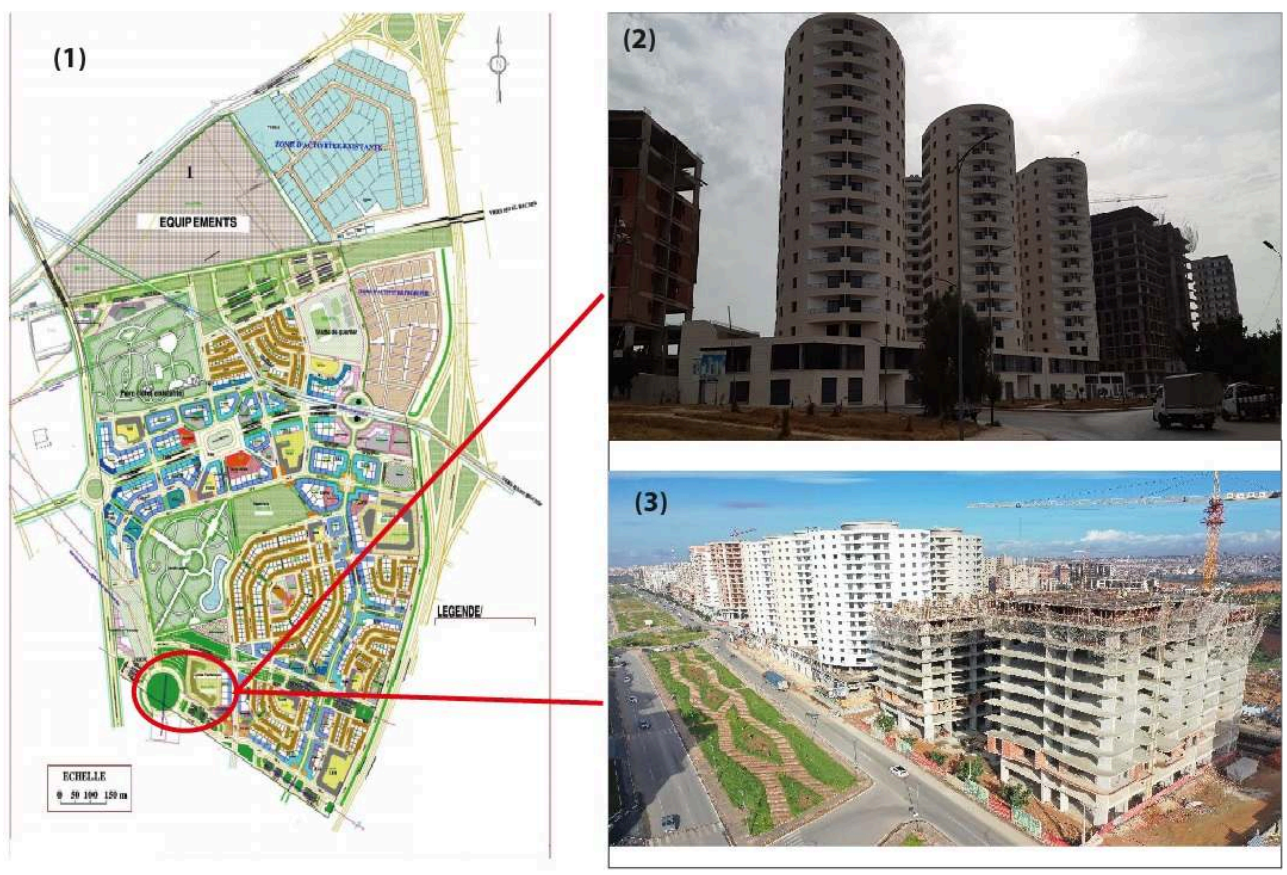

Sources : (1) plan parcellaire du POS51 (1998), mairie de Bir El Djir

(2) résidence «ElHadja Fatima », photo prise par les auteures (2020)

(3) photo aérienne de la résidence «Les Zianides », Ben Melissa Promotion (2019)

À travers l'exemple de Bir El Djir, on constate que le phénomène de détournement prend plusieurs formes et s'opère dans des situations variées. Les enjeux en matière d'aménagement et d'appropriation foncière dans l'espace périurbain s'articulent, essentiellement, autour des POS. Toute opération d'affectation d'un lot de terrain est soigneusement déclarée en suivant les procédures légales. L'administration trouve toujours le moyen pour légaliser les différentes actions touchant le foncier ce qui apporte une certaine légitimité sociale et politique à ce que nous, chercheurs, qualifierons de détournement ou pillage de la ressource foncière. Toutes les infractions commises sur les réserves foncières sont, officiellement, justifiées par la nécessité publique. Officieusement, ces processus d'acquisition et de réaffectation des sols sont largement reconnus comme des procédures de complaisance et d'arrangement avec certains administrateurs et promoteurs immobiliers. Selon un interlocuteur à la Direction de l'Urbanisme et de la Construction de la wilaya d'Oran «Lors de l'élaboration des PDAU et des POS, on assiste, dans tous les niveaux des administrations foncières, à des alliances de toute nature mais pour un seul objectif, celui de l'accaparement de la ressource foncière... tout le monde y trouve son compte : les promoteurs immobiliers, les hommes d'affaires, les élus, les collectivités locales et autres. Tout parait légal sur les dossiers, mais tout le monde sait que des arrangements, en coulisses, sont à l'origine de certaines affectations de terrains ".

Dans la périphérie oranaise, on assiste, aujourd'hui, à un foisonnement de promotions immobilières de haut standing (fig. 10), qui répondent à des critères de luxe recherchés par une clientèle fortunée, parfois non résidente à Oran, et qui ne règlent certainement pas la crise du logement. 
Figure 10. Quelques affiches publicitaires de promotions immobilières de haut standing dans la périphérie oranaise.
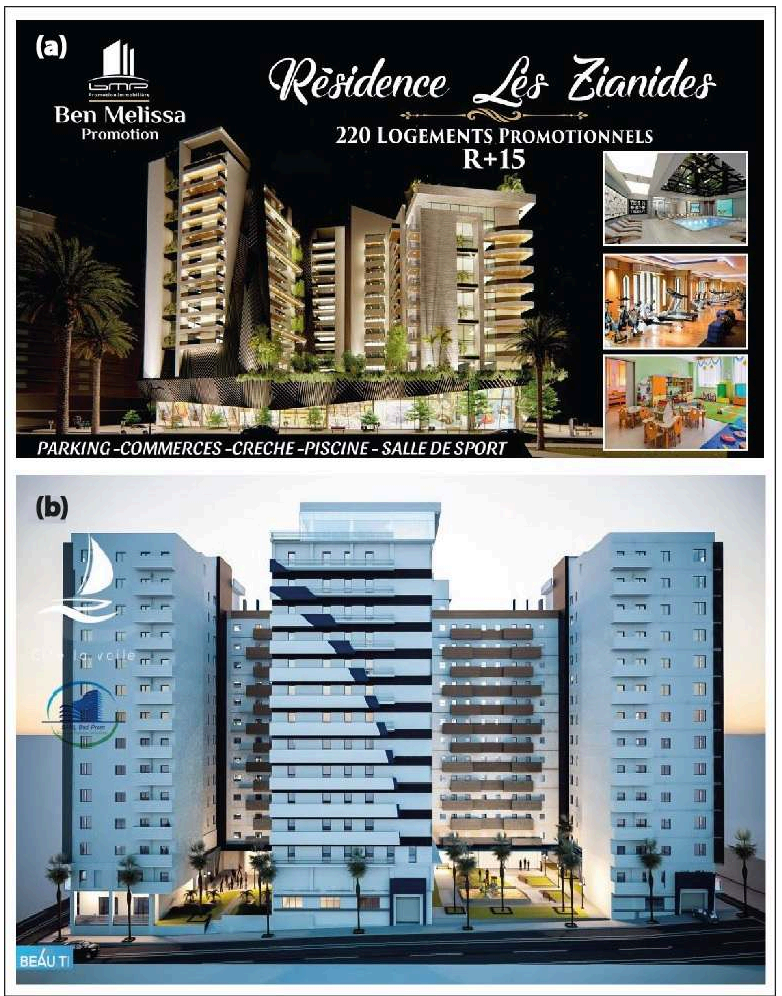

(a) Promotion immobilière «Les Zianides », Boulevard des Lions, Commune de Bir El Djir

(b) Promotion immobilière "Cité la voile », Canastel, Commune d'Oran

Source : affiches publicitaires Ben Melissa Promotion \& SARL BAD PRO

\section{Conclusion générale}

La décomposition et recomposition fonctionnelle de l'espace agricole périurbain d'Oran est la résultante des politiques agricoles et urbaines engagées par les pouvoirs centraux au lendemain de l'indépendance. Contrairement aux objectifs recherchés, la politique agricole a créé la distanciation entre l'exploitant et le statut juridique de l'exploitation (l'État reste le propriétaire des meilleures terres agricoles du pays). Cette situation a généré des pseudos agriculteurs en manque d'initiatives et de projet agricole déterminé. Leur sentiment d'insécurité foncière et de non appartenance à la terre les pousse à cumuler d'autres fonctions non agricoles ou à sous-louer les terres que l'État leur a confiées. L'agriculture souffre alors du manque d'investissement humain et les terres agricoles jouxtant les grandes agglomérations deviennent un réservoir à la disposition des demandes des autres secteurs : habitat urbain, industrie, réseaux de circulation et de communication (routes, chemins de fer, etc.). La crise du secteur de l'agriculture est bien résumée par l'expression "un colosse aux pieds d'argile » de Yazid Ferhat à l'occasion des Assises Nationales sur l'Agriculture du 23 avril 2018 sous le thème "L'agriculture au service de la souveraineté nationale ».

Le PDAU et le POS n'ont pas permis de contenir l'étalement urbain et de préserver les terres agricoles contre la machine à bâtir. Un tel constat vient confirmer, d'une part, l'existence d'un décalage entre les discours et les ambitions des acteurs politiques et la 
réalité du terrain, et d'autre part les limites des instruments d'urbanisme face à la dynamique du contexte démographique, social et économique (Boumaza, 2006) et les défaillances de l'administration avec tous ses services responsables de la gouvernance foncière (Wilaya, les communes, la direction des services agricoles, les domaines, les agences foncières, etc.).

Ces faiblesses ont agi comme des opportunités pour des acteurs privés et publics de recourir à des pratiques illicites et à des dépassements pour le détournement d'usage du foncier agricole. La Direction des Services Agricoles affiche souvent une fragilité et un laisser faire lors de la validation des choix des futurs terrains à urbaniser par le PDAU ; son retrait ne serait-il pas expliqué par l'absence d'un vrai projet agricole de la zone périurbaine de la métropole oranaise?

L'agriculture périurbaine, qui reste une problématique d'actualité pour les chercheurs et est discutée dans les différents articles scientifiques ou colloques internationaux (Charvet, 2013; Semmoud, Ladhem, 2015 ; Colloque international de Valenciennes, $2018^{14}$ ), figure rarement dans les programmes des élus ou dans les plans d'action de la wilaya ou encore dans les projets futurs des différents agriculteurs qui résident encore dans le périurbain. La déficience de la production agricole des terres fertiles du périurbain (malgré la reconnaissance du potentiel agricole des sols) met la Direction des Services agricoles en position de faiblesse par rapport aux autres acteurs comme les promoteurs immobiliers qui construisent à la place de l'État, ou de la wilaya qui, sous la pression des ministères et de la société civile, doit répondre aux besoins urgents de son territoire en matière de logements et d'infrastructures.

Même si les terres agricoles sont importantes pour assurer la sécurité alimentaire du pays, est-il possible de les protéger contre l'urbanisation? Et si oui, comment? Il apparaît que l'urbanisation ne pourrait être évitée au vu de la croissance des besoins en logements et en infrastructures en ville, par contre son rythme pourrait être substantiellement réduit si un ensemble de mesures bien pensées et coordonnées dans le temps et dans l'espace était mis en œuvre. La refonte des instruments d'aménagement et d'urbanisme privilégiant une démarche intégratrice et stratégique s'impose.

61 Dans ce sens, il est important de retenir que «La terre est une source non renouvelable et on ne peut pas l'importer. Il y a lieu de la préserver. Mais aussi ne pas rééditer les erreurs des années 1980 et 1990, durant lesquelles des centaines de milliers d'hectares de terres agricoles ont été transformés en foncier industriel et urbanisable, sans résultats palpables aujourd'hui en termes de développement économique " (Ahmed Ali, 2013).

Les espaces agricoles périurbains seraient à insérer dans un projet de territoire partagé par le ministère de l'agriculture, les différents élus, les associations civiles ainsi que les agriculteurs eux-mêmes; ils devraient être une issue et une solution pour recréer ce lien fonctionnel entre les espaces agricoles périurbains et l'agglomération. Cela permettrait de mettre en place un pacte et des moyens de négociation en impliquant un grand nombre de partenaires (l'État, les agriculteurs, les consommateurs, les associations civiles, les élus, les investisseurs dans l'agriculture, etc.). Si l'espace agricole périurbain est doté d'un vrai projet de territoire délimité, alors il «cesse d'être un vide du point de vue urbain » (Serrano, 2005).

Dans cette perspective, il est temps que la paysannerie algérienne redevienne un acteur actif du territoire, cela mènera l'État (planificateur) à adopter une vision plus large et plus globale des territoires métropolitains. En tant qu'acteur central du pouvoir, l'État 
doit renoncer à la vision sectorielle qui positionne toujours l'agriculture derrière la ville et l'industrie. Il est inutile de débloquer des enveloppes financières colossales pour assister l'agriculture algérienne si la sécurité foncière est inexistante et que le facteur principal de production, la terre, est perpétuellement, amputée pour combler les besoins de la ville et des perspectives économiques hors agriculture.

En somme, le dilemme ville/campagne ne peut pas être résolu du jour au lendemain, car le foncier, leur dénominateur commun, reste une équation complexe et à variables multiples. Étant le support de toute vie et autour duquel l'ensemble des rapports sociaux se tissent, le foncier a une très forte prégnance sur les configurations des territoires. Dans le contexte algérien, les rapports de pouvoir entre la ville et l'agriculture sont des plus asymétriques et se traduisent par une forme de domination, excessive, de la ville sur l'agriculture en accaparant le sol agricole pour la concrétisation de ses projets sous couvert de l'utilité publique, et par contournement de la règlementation. Le foncier ne serait-il pas alors une boîte noire de l'économie et de la politique où la convoitise, la spéculation ou la connivence s'invitent? Aussi, le foncier est un objet d'étude stratégique et sensible car il est par définition une question politique et de politique.

\section{BIBLIOGRAPHIE}

AHMED ALI A. (2013), « Grande pression sur le foncier agricole, 20000 hectares sacrifiés depuis 2010 au profit de l'urbanisation ", in El Watan », édition du 6 mai 2013 (Article rédigé par Samira Imadalou).

AMEURM. (1993), FÈS... ou l'obsession du foncier, URBAMA Tour, France.

BENDJELID A. (1998), «La fragmentation de l'espace urbain d'Oran (Algérie). Mécanismes, acteurs et aménagement urbain », Insaniyat, 5, pp. 61-84.

BENDJELID A., HADEID M., MESSAHEL A. \& TRACHE S.M. (2004), « Différenciations sociospatiales dans les nouveaux espaces urbanisés d'Oran », Insaniyat 23-24, pp. 7- 44.

BENKADA S. (2001), « Un demi-siècle d'extension de l'espace périphérique oranais, à travers quelques exemples de politiques d'urbanisation (1948-1998) », Insaniyat, 13, pp. 95-104.

BESSAOUD O. (2019), Projet d'appui à l'initiative ENPARD MEDITERRANEE, Rapport de synthèse sur l'agriculture en Algérie.

BOUMAZA N. (dir.) (2006), Villes réelles, villes projetées. Villes maghrébines en fabrication, Paris, Maisonneuve \& Larose, France.

CHARVET J.P. (2013), « Avant-Propos - Agriculture dans la ville, agriculture pour la ville : acteurs, pratiques et enjeux », Bulletin de l'Association de Géographes Français, 90, 3, pp. 273-275

COTE M. (1993), L’Algérie ou l'espace retourné, Média-plus, Constantine, Algérie. 
DALIGAUX J. (2001), « La périurbanisation en Provenance : visages d'hier et d'aujourd'hui, interrogations pour demain. Le cas du Var et du Bouche-du-Rhône », Géocarrefour, 76, 4, pp. 289-302.

DARLY S., TORRE A. (2008) : « Conflits liés entre aux espaces agricoles et périmètres de gouvernance en Ile-en-France ", Géocarrefour, 83, 4, pp. 307-319.

DURAND-LASSERVE A., TRIBILLON J.F. (1983), « La production foncière et immobilière dans les pays en développement ", Hérodote, 31 : l'implosion urbaine, pp. 9-36.

GHAOUTI S. (2014), « Le traitement de la propriété dans les textes fondamentaux algériens ", Revue algérienne des sciences juridiques, économiques et politiques, numéro spécial, mars, pp. 9-30.

GUERROUDJ T. (2004), « Les enjeux de l'organisation de l'agglomération oranaise », Insaniyat, 23-24, pp. 45-61.

HAMDANI H. (2018), « Déclassement des terres agricoles : "Un impératif extrême" », in Le Soir d'Algérie, édition du $1^{\mathrm{er}}$ décembre 2018 (article rédigé par Ry. N.).

HOUDART M., LOUDIYI S. \& GUERINGER A. (2012), « L'adaptation des agriculteurs au contexte périurbain. Une lecture des logiques agricoles à partir du cas de Billom-Saint-Dier (Auvergne) », Norois, 224, 3, pp. 35-47.

JARRIGE M., JOUVE A.M. \& NAPOLEONE C. (2003), « Et si le capitalisme patrimonial foncier changeait nos paysages quotidiens? », Courrier de l'Environnement de l'INRA, 49, pp. 13-28.

JOUVE A.M., VIANEY G. (2012), « Le foncier, une ressource territoriale difficile à construire en périurbain ", Économie rurale, 330-331, 4-5, pp. 27-41.

KADRI Y., MADANI M. (2015), « L'agglomération oranaise (Algérie) entre instruments d'urbanisme et processus d'urbanisation », EchoGéo, 34, http://echogeo.revues.org/14386 KETTAF F. (2013), La fabrique des espaces publics : Conceptions, formes et usages des places d'Oran (Algérie), Thèse de doctorat en géographie, Université Paul Valéry, Montpellier, $467 \mathrm{p}$.

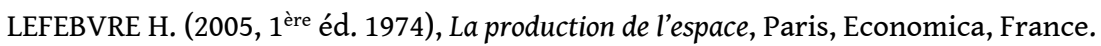

MAACHOU H.M. (2008), « Conflits d'usages et dynamiques spatiales ; les antagonismes dans l'occupation des espaces périphériques des grandes villes en Algérie, cas d'Oran ", Bulletin des sciences géographiques, 21, Institut national de cartographie et de télédétection, pp. 23-28.

MAACHOU H.M., OTMANE T. (2016), «L'agriculture périurbaine à Oran (Algérie) : diversification et stratégies d'adaptation ", Cahiers Agricultures, 25.

MADJARIAN G. (1991), L'invention de la propriété: de la terre sacrée à la société marchande, L'Harmattan, France.

MARIÉ M., VIARD J. (1977), La campagne inventée ou ce qu'il advient des rapports entre les paysans, leurs communautés et l'environnement urbain dans quatre villages d'un pays de moyenne Provence, Actes Sud, France.

MARTIN S., BERTRAND N. \& ROUSIER N. (2006), « Les documents d'urbanisme, un outil pour la régulation des conflits d'usage de l'espace agricole périurbain ", Géographie Economie Société, 8, 3, pp. 329-350.

MISSOUMI M.A., HADEID M. \& DESPONDS D. (2019), « Jeux d'acteurs et fragilisation de l'agriculture périurbaine dans l'agglomération d'Oran (Algérie) », Études caribéennes, 43-44. 
MUTIN G. (1977), La Mitidja, décolonisation et espace géographique, Alger-Paris, CNRS, Office des publications universitaires.

NEMOUCHI H. (2009), La question du foncier agricole en Algérie : pratiques foncières/pratiques sociales. Le cas de Salah Bouchaour (nord-est algérien), Thèse de Doctorat, Université de Caen Normandie, $343 \mathrm{p}$.

NEMOUCHI H. (2011), « Pratiques sociales et problèmes fonciers en Algérie », in ELLOUMI M., JOUVE A.-M., NAPOLÉONE C. \& PAOLI J.-C. (éd.), Régulation foncière et protection des terres agricoles en Méditerranée, Options Méditerranéennes, 66, pp. 127-143.

OFFICE NATIONALE DES STATISTIQUES (1966), Recensement Général de la Population et de l'Habitat (RGPH), Algérie.

OFFICE NATIONALE DES STATISTIQUES. (2008), Recensement Général de la Population et de l'Habitat (RGPH), Algérie.

OMARI C., MOISSERON J.-Y. \& ALPHA A. (2012), « L'agriculture algérienne face aux défis alimentaires. Trajectoires historiques et perspectives », Revue Tiers Monde, 210, 2, pp. 123-141.

PELTIER C. (2010), « Agriculture et projet urbain durables en périurbain : la nécessité d'un réel changement de paradigme ", Vertigo. La revue électronique en science de l'environnement, 10, 2.

PERRIN C., SOULARD C.T. \& CHIA E. (2016), « La gouvernance du foncier agricole périurbain : entre planification urbaine et projets de développement ", Revue d'Economie Régionale et Urbaine, 4 , pp. 713-736.

POULOT M. (2014), « Agriculture et acteurs agricoles dans les mailles des territoires de gouvernance urbaine : nouvelle agriculture, nouveaux métiers? », Espaces et Sociétés 158, 3, pp. 13-30.

PROST B. (1991), « Du rural au péri-urbain : conflit de territoire et requalification de l'espace », Revue de géographie de Lyon, 66, 2, Mutations économiques et requalifications territoriales, pp. 96-102.

RENARD V. (2003), Préface - Bouleversements fonciers en Méditerranée, CIHEAM, Karthala, France.

ROUGE L. (2018), « Périurbanisation », Géoconfluences, http://geoconfluences.ens-lyon.fr/ informations-scientifiques/a-la-une/notion-a-la-une/notion-a-la-une-periurbanisation

SEMMOUD B., LADHEM A. (2015), « L'agriculture périurbaine face aux vulnérabilités foncières en Algérie », Territoire en mouvement 25-26, https://journals.openedition.org/tem/2845

SERRANO J. (2005), « Quel équilibre entre urbanisation et préservation des espaces agricoles périurbains ? Le cas d'une agglomération moyenne ", Développement durable et territoires, Dossier $\mathrm{n}^{\circ} 4$.

SERRANO J., VIANEY G. (2011), « Consommation d'espace agricole et relations entre acteurs privés et publics : un management en faveur de l'artificialisation », Norois, 221, 4, pp. 111-124.

TRACHE S.M. (2010), Mobilités résidentielles et périurbanisation dans l'agglomération oranaise, Thèse de doctorat, Université d'Oran, $202 \mathrm{p}$.

UNITED NATIONS (2014), The 2014 Revision, Department of Economic and Social Affairs/Population Division World Urbanization Prospects.

ZEGHICHE A. (2007), « Le foncier agricole en Algérie (XIX-XX ${ }^{\text {ème }}$ siècle) : discours et pratiques sociales ", Enquêtes Rurales, $n^{\circ} 11$ : Société, pouvoirs et politique dans les campagnes, Cahiers de la Maison de la Recherche en Sciences Humaines, Presses Universitaires de Caen, France, pp. 107-126. 
ZOUANI R. (2016), Les instruments d'urbanisme entre textes législatifs et réalité pratique. Le cas des pos a Bir El Djir, Mémoire de fin d'études, Université d'Oran 2.

\section{NOTES}

1. PDAU : Plan Directeur d'Aménagement et d'Urbanisme.

2. POS : Plan d'Occupation du Sol.

3. GUO : c'est le périmètre de développement de l'agglomération oranaise délimité par le Plan Directeur d'Aménagement et d'Urbanisme en 1998 ; il est composé de quatre communes: Oran, Bir El Djir, Es Sénia et Sidi Chahmi.

4. APC : Assemblée Populaire Communale (la Mairie).

5. CAPAM : Coopérative Agricole de Production des Anciens Moudjahidines.

6. La loi 87-19 du 8 décembre 1987 réforme le mode d'exploitation des terres agricoles du domaine public. La nue-propriété de la terre reste à l'État, mais celui-ci accorde aux collectifs d'exploitants un droit de jouissance, à titre perpétuel et transmissible, sur ces mêmes terres. En contrepartie, les collectifs d'exploitants verseront à l'État une redevance. Les exploitants acquièrent également un droit de pleine propriété sur tous les biens d'exploitation autres que la terre. D'autre part, la loi stipule que les revenus des producteurs découleront exclusivement des résultats de la production; l'État ne garantira plus un salaire aux travailleurs agricoles, et il ne devrait plus intervenir dans la gestion des exploitations agricoles.

7. La loi 10-03 du 15 août 2010 a pour objet de fixer les conditions et les modalités d'exploitation des terres agricoles du domaine privé de l'État. Cette exploitation se réalise au moyen d'une concession, acte par lequel l'État consent, à une personne physique de nationalité algérienne, ciaprès désignée "exploitant concessionnaire", le droit d'exploiter des terres agricoles du domaine privé de l'État ainsi que les biens y rattachés, sur la base d'un cahier des charges fixé par voie réglementaire.

8. Selon l'ONS, au cours de l'année 2018, nous assistons à un volume de naissances vivantes atteignant 1.038.000. Le taux brut de natalité est estimé à $24,39 \%$ en 2018 .

9. Recensement Général de la Population et de l'Habitat 1966.

10. Recensement Général de la Population et de l'Habitat 2008.

11. Malgré l'ancienneté du présent chiffre, l'intérêt d'utiliser les données du tableau $n^{\circ} 5$ réside dans l'analyse du processus administratif d'affectation des surfaces agricoles à l'urbanisation.

12. Décret exécutif $n^{\circ} 11-237$ du 9 juillet 2011 portant déclassement de parcelles de terres agricoles affectées pour la réalisation de logements publics et des équipements d'accompagnement dans certaines wilayas et le décret exécutif $n^{\circ}$ 12-370 du 24 octobre 2012 portant déclassement de parcelles de terres affectées pour la réalisation de projets publics de développement.

13. Une centaine d'hectares de foncier détournés par des oligarques à Bir El-Djir (Réflexion 17/8/2019). Bir el djir (Oran): 48 détournements de foncier recensés (Algérie $360^{\circ}$, site internet d'informations algérien-12/2/2017); Les «vraies affaires» de prédation foncière (Ouest Tribune, 13 mai 2019).

14. Rencontres interdisciplinaires Sociétés-Environnement sous le thème «Terra Mater, Les sols convoités ", 2018, Université de Valenciennes et du Hainaut Cambrésis. 


\section{RÉSUMÉS}

Aujourd'hui, la périurbanisation est reconnue comme un phénomène mondial animée par différents déterminants (poussée démographique, développement industriel, crise de la ruralité, etc.). En effet, les territoires périurbains sont des espaces qui reçoivent l'excédent de la croissance démographique urbaine et qui accueillent les nouveaux projets d'aménagement et de développement (promotions immobilières, implantations de zones d'activités diverses, développement des réseaux de communication, maintien ou développement de certaines agricultures, etc.). Dans ces zones périurbaines où se mêlent l'urbain et le rural, les vives tensions autour de la terre s'exacerbent. La question de la maitrise et de la gestion du foncier constitue l'un des dossiers les plus lourds et les plus complexes : si le foncier est la clé de voûte de l'activité socioéconomique, s'il se situe à la croisée des enjeux et des orientations d'aménagement, sa complexité freine souvent la définition des stratégies susceptibles d'y répondre. C'est dire que le foncier a une très forte prégnance sur les configurations et les évolutions des territoires urbains et ruraux et sur les rapports villes-campagnes.

L'objectif du présent article est de saisir les processus de recomposition de territoires ruraux algériens depuis l'indépendance, d'expliquer les mécanismes d'évolution en œuvre et de décrypter les stratégies développées par les acteurs publics pour transformer les terres agricoles en terres constructibles (terrain à urbaniser ou pour urbanisation futur) dans les espaces périurbains des grandes villes algériennes. Pour donner plus de sens et de réalité aux dynamiques du monde rural algérien, l'investigation porte sur l'espace périurbain d'Oran, deuxième ville d'Algérie et métropole régionale de l'Oranie; elle vise à apporter quelques éléments d'éclairage sur certains des mécanismes de réappropriation / réaffectation du sol agricole au profit de projets urbains.

Nowadays, peri-urbanization is recognized as a global phenomenon prompted by different determinants (demographic growth, industrial development, rurality crisis, etc.). In fact, periurban territories are spaces which receive the excess of the urban demographic growth as well as they welcome the new planning and development projects (housing promotions, establishment of various activity zones, development of communication networks, and maintenance or development of certain types of agriculture, etc.).

In such peri-urban areas where urban and rural mingle, strong tensions around the land are exacerbated. The matter of control and management of land is one of the heaviest and most complex issues: if land is the keystone of socioeconomic activity and if it is the crossroads of issues and development orientations, its complexity often hinders the definition of strategies that can probably resolve them. This means that land has such a strong influence on the configurations and developments of urban and rural territories as on city-countryside relationships.

The objective of this article is to understand the recomposition processes of Algerian rural territories since the independence (1962), to explain the outstanding mechanisms of evolution and to decipher the strategies developed by public actors in order to transform agricultural land into building ones (land to be urbanized or for future urbanization) in the peri-urban spaces of large Algerian cities. To give more meaning and reality to the Algerian rural world dynamics, the investigation focuses on the peri-urban area of Oran, the second city of Algeria and regional metropolis of Orania; it aims to shed some light on some of the mechanisms for the reappropriation/reallocation of agricultural land for the benefit of urban projects. 
INDEX

Mots-clés : foncier agricole, périurbanisation, instrument d'urbanisme, stratégies d'acteurs, détournement d'usage, Oran, Algérie

Keywords : agricultural land, peri-urbanization, urban development instrument, stakeholder strategies, diversion of use, Oran, Algeria

\section{AUTEURS}

\section{HAYETTE NEMOUCHI}

Géographe et chercheure au Centre de Recherche en Anthropologie Sociale et Culturelle - Oran, Algérie, hayette.nemouchi@gmail.com

\section{ANISSA ZEGHICHE}

Laboratoire de Recherche « Ressources Naturelles et Aménagement », Université Badji MokhtarAnnaba-Algérie, boukhemisk@yahoo.com 